\title{
Assessing placental function across gestation: a multi-institutional study of BOLD-MRI for the prediction of adverse pregnancy outcomes
}

\author{
Matthias Schabel \\ Oregon Health \& Science University

\section{Victoria Roberts} \\ Oregon National Primate Research Center
}

\section{Karen Gibbins}

Oregon Health and Science University

\section{Monica Rincon}

Oregon Health and Science University

Jessica Gaffney

Oregon Health and Science University

\section{Aaron Streblow}

Oregon Health and Science University

\section{Adam Wright}

Oregon Health and Science University https://orcid.org/0000-0001-9524-2210

Jaime Lo

Oregon Health \& Science University

Byung Park

Oregon Regional Primate Center

Christopher Kroenke

Oregon Health \& Science University https://orcid.org/0000-0001-7398-3632

Kathryn Szczotka

University of Utah

Nathan Blue

University of Utah

Jessica Page

University of Utah

Kathy Harvey

University of Utah

Michael W. Varner

University of Utah School of Medicine

\section{Robert Silver}


University of Utah

Antonio Frias ( $\square$ friasa@ohsu.edu )

ONPRC/OHSU

\section{Article}

Keywords: Maternal-fetal Interactions, Fetal Development, Placental Dysfunction, Pregnant Non-human Primate, Maternal-fetal Oxygen Transport

Posted Date: April 26th, 2021

DOl: https://doi.org/10.21203/rs.3.rs-406266/v1

License: (9) This work is licensed under a Creative Commons Attribution 4.0 International License. Read Full License 
1 Assessing placental function across gestation: a multi-institutional study of BOLD-MRI for

2 the prediction of adverse pregnancy outcomes.

3

4 Matthias C Schabel, $\mathrm{PhD}^{1 \# \star}$, Victoria HJ Roberts, $\mathrm{PhD}^{2 \#}$, Karen J Gibbins, MD ${ }^{3 \#, ~ M o n i c a ~}$

5 Rincon, $\mathrm{MD}^{3}$, Jessica E Gaffney, $\mathrm{BS}^{2}$, Aaron D Streblow, $\mathrm{BS}^{2}$, Adam M Wright, $\mathrm{BS}^{2}$, Jamie $\mathrm{O}$

6 Lo, $\mathrm{MD}^{3}$, Byung Park, $\mathrm{PhD}^{4}$, Christopher D Kroenke, $\mathrm{PhD}^{1,5}$, Kathryn Szczotka, $\mathrm{MPH}^{6}$, Nathan R

7 Blue, $\mathrm{MD}^{6}$, Jessica M Page, $\mathrm{MD}^{6}$, Kathy Harvey, $\mathrm{BS}^{6}$, Michael W Varner, $\mathrm{MD}^{6}$, Robert M Silver,

$8 \mathrm{MD}^{6}$, Antonio E Frias, $\mathrm{MD}^{2,3 *}$

9

$10{ }^{1}$ Advanced Imaging Research Center, Oregon Health and Science University (OHSU)

11 2Division of Reproductive and Developmental Sciences, Oregon National Primate Research

12 Center (ONPRC), OHSU

$13{ }^{3}$ Department of Obstetrics and Gynecology, OHSU

$14 \quad{ }^{4}$ Biostatistics Shared Resource, Knight Cancer Institute, OHSU

$15 \quad{ }^{5}$ Division of Neuroscience, ONPRC, OHSU

$16{ }^{6}$ Department of Obstetrics and Gynecology, University of Utah

$17 \quad{ }^{*}$ Corresponding authors

18 \#Joint first authors 


\section{Abstract}

The placenta is a remarkable organ that coordinates and regulates maternal-fetal interactions during pregnancy to optimize fetal development. A host of obstetric complications are associated with placental dysfunction, and existing methods for evaluating in vivo placental function fail to reliably detect at-risk pregnancies prior to maternal or fetal morbidity. Although routinely used as a monitoring tool, the predictive power of ultrasound for identifying compromised pregnancies is poor. Recent preclinical studies performed in our laboratory, using blood oxygen-level dependent magnetic resonance imaging (BOLD-MRI) in the pregnant nonhuman primate (NHP), established a strong correlation between placental T2* values and maternal-fetal oxygen transport. Here we extend this work to a large, longitudinal, two-site study of quantitative in vivo T2* mapping in human pregnancies across 11 to 38 weeks gestation to characterize the evolution of placental oxygenation in uncomplicated pregnancies and to elucidate the relationship between aberrant placental T2* and adverse obstetric outcomes attributable to placental dysfunction. This methodology has high discriminatory power and strong potential diagnostic utility.

\section{Introduction}

The fundamental role of the placenta in fetal development, pregnancy morbidity, and neonatal, pediatric, and even lifelong health is indisputable (1-7). Aberrant placental development has been linked to virtually every adverse obstetric outcome, including abnormalities in fetal growth, preeclampsia, preterm labor, and stillbirth (4, 8-17). During pregnancy, the placenta supplies oxygen and critical nutrients required for fetal growth, removes waste products from the fetal circulation, protects the fetus from environmental toxins and infections, produces pregnancyspecific hormones, and mediates communication between the fetus and the mother to coordinate maternal physiologic adaptations and fetal development (18-20). The regulation of all of these processes changes dynamically across gestation to ensure appropriate maternal resource allocation to meet fetal growth demands. Although mechanisms regulating normal placental growth and development are incompletely understood, the central role of the placenta

48 The inability to longitudinally sample placental tissue during gestation constitutes a significant limitation for the study and assessment of placental development in human pregnancies. 
development across gestation and assess placental function and health in vivo is a crucial component in the identification of pregnancies at risk for adverse obstetric and neonatal outcomes.

Obstetric imaging, predominantly with ultrasound (US), is a mainstay of clinical care for identification of fetal anomalies and detection of aberrant fetal growth (21-25). Uterine artery velocimetry has been studied as a potential predictor of preeclampsia and fetal growth restriction (FGR), and has modest predictive power for severe, early onset phenotypes of both $(22,24,26)$, but it performs poorly in predicting later onset morbidity due to placental dysfunction, possibly because it measures resistance to blood flow (impedance) in the umbilical artery rather than focusing on perfusion of the placenta itself (27). Similarly, fetal umbilical artery Doppler ultrasound is used to risk stratify pregnancies suspected to have FGR based on ultrasound fetal biometry. Observation of abnormal blood flow via umbilical artery Doppler assessment, particularly absent or reversed diastolic blood flow, is clearly associated with adverse perinatal outcomes $(28,29)$. However, its principal utility is in antenatal surveillance to guide hospitalization and timing of delivery after the diagnosis of FGR has already been established by ultrasound-based biometry, not in prediction of incipient FGR. Although it is clear that profoundly abnormal umbilical artery blood flow in the setting of FGR is associated with adverse perinatal outcome (28), it is not a direct measure of placental function and it can be normal in some cases of severe placental insufficiency. Despite its widespread use, the utility of screening ultrasound as a tool for identification of pregnancies at risk for adverse outcomes remains limited.

Magnetic resonance imaging (MRI) has been used during pregnancy for decades, primarily to assess fetal abnormalities via anatomic imaging. Recently, the NIH Human Placenta Project stimulated the development and application of a number of innovative MRI techniques, intended to enable in vivo assessment of placental function during pregnancy (30). Early work by Sorensen and colleagues (31) observed the presence of spatial heterogeneity in T2*-weighted MRI of the placenta, and found that this heterogeneity was decreased by maternal hyperoxia. It is well-known that $\mathrm{T}^{*}$-weighted images are sensitive to changes in the relative levels of oxyhemoglobin and deoxyhemoglobin via the blood oxygenation level dependent (BOLD) effect, which forms the basis of functional MRI (fMRI) studies of the brain (32). Consideration of the specifics of the anatomy and physiology of hemochorial placentas led us to hypothesize that quantitative $\mathrm{T} 2^{*}$ measurements could be used to assess placental perfusion and maternal-fetal oxygen transport. Subsequent work performed by our group in pregnant nonhuman primates 
(NHPs), combining dynamic contrast enhanced (DCE-) MRI with quantitative T2* mapping (33, 34) proved that the observed heterogeneity in placental T2* arises from spatial gradients in maternal placental blood (MPB) oxygen saturation within functional lobules. We then developed a simple model based on relevant physiological parameters of the placenta that accounts for these spatial variations. Highly oxygenated maternal blood delivered to the placenta via spiral arteries has large ${ }^{*}{ }^{*}$ values that decrease continuously toward the placental lobular margins as oxygen from the MPB is extracted by the villi and transported to the fetus. This research demonstrates that $\mathrm{T}^{*}$ relaxometry provides a measure of the critical balance between maternal supply of oxygen to the fetal vasculature and fetal uptake of the supplied oxygen. Recent NHP studies from our group have further shown that anomalous baseline T2* values are correlated with placental dysfunction in cases of fetal growth restriction as well as secondary to perturbations with prenatal alcohol exposure or Zika virus infection (35-37).

Placental T2* has been measured throughout gestation in both uncomplicated human pregnancies and human pregnancies with adverse outcomes by a number of groups (38-44). These studies verified that placental T2* decreases with gestational age, consistent with results from NHP studies and with the expected effect of increased fetal oxygen demand with growth. Given the direct association between $\mathrm{T}^{*}$ and maternal-fetal oxygen transport, it is conceivable that this non-invasive MRI measure could be applicable to clinical studies of placental function. However, the paucity of longitudinal data, incorporation of specialized image acquisition or analysis procedures, and minimal experience comparing data between sites limit our current ability to extend T2* measurements to large-scale studies.

105 Based on preliminary evidence in NHP and human pregnancies, we hypothesized that placental 106 T2* values in pregnancies complicated by clinical placental dysfunction would be lower for 107 gestational age compared to uncomplicated pregnancies as a result of inadequate maternal 108 oxygen supply to the placenta. The primary objective of this work was to test this hypothesis by conducting a prospective, longitudinal, two-site MRI study in 316 pregnant women, with intentional population enrichment for obstetric morbidity due to placental dysfunction. A secondary objective was to establish whether data acquired at two different institutions, using compatible scanner hardware and MRI protocols and analyzed by uniform post processing, would demonstrate sufficient data consistency to support conducting larger multi-institutional studies of this non-invasive imaging method to identify and assess placentally-mediated adverse pregnancy outcomes. 


\section{Results}

118 Participant and study demographics

119 Details of participant enrollment and study completion at the two study sites are presented in the flow chart in Figure 1. Demographics and maternal characteristics are detailed in Table 1. MRI data of adequate quality to perform T2* analysis were acquired in 797 imaging studies from 316 individual study participants (450 scans from 179 participants at OHSU, 347 scans from 137 participants at Utah). At least one usable MRI scan was obtained from $86 \%$ of participants who were consented ( $88 \%$ at OHSU, $83 \%$ at Utah), with all three scans completed in $66 \%$ of these patients, two scans in $20 \%$, and a single scan in $14 \%$. Of these studies, 700 had usable hemoglobin data (426 at OHSU, 274 at Utah) and 432 had usable SpO2 measurements (252 at OHSU, 180 at Utah). 352 usable quantitative T1 measurements were also obtained in 156 of the OHSU participants.

Pregnancy outcomes

Out of 316 participants, 198 (62.6\%) were classified as uncomplicated pregnancies (UN), 70 $(21.8 \%)$ were classified in the primary adverse (PA) outcome group, and $48(15.2 \%)$ were classified in the secondary abnormal (SA) outcome group, as defined in the Methods. Within primary adverse outcome group, the most commonly observed component was preeclampsia with severe features $(40.0 \%)$ followed by gestational hypertension $(31.4 \%)$, and small for gestational age (SGA, 20.0\%) (Table 2). Both severe pre-eclampsia and SGA were more prevalent in the OHSU cohort than the Utah cohort, but the observed differences were not statistically significant on chi-square test. $35.7 \%$ of those with the primary adverse outcome delivered prior to 37 weeks, our threshold for prematurity.

\section{Birthweight statistics}

140 Median birthweight percentile (computed using the tables from Oken (45)) was 48.2 in UN 141 pregnancies, 32.7 in PA pregnancies, and 48.8 in the SA pregnancies. There was a significant 142 difference in birthweight percentile between UN and PA outcome groups $(p=0.017)$ but not 143 between UN and SA ( $p=0.28$ ). When stratified by study site, birthweight percentiles for UN, PA, 144 and SA were 50.5, 30.3, and 49.3 at OHSU, and 42.0, 36.1, and 46.5 at Utah, respectively. 145 Birthweight was significantly lower in PA vs. UN outcome groups in the OHSU cohort $(p=0.023)$ 146 but not at Utah $(p=0.42)$. There was no difference between UN and SA outcome groups at 147 either site (OHSU $p=0.74$, Utah $p=0.51)$. 
149 Figure 2 shows representative anatomic T2-weighted HASTE (left column) and quantitative T2*

150

151

152

153

154

155

156

157

158

159

160

161

162

163

164

165

166

167

168

169

170

171

172

173

174

175

176

177

178

179 maps (right column) acquired in two study participants, matched for gestational age at time of scan. Placental regions of interest (ROIs) are superimposed on the T2* maps (blue dashed lines). The upper row in the figure shows a UN pregnancy at 232 days of gestation with median placental T2* $(=51 \mathrm{~ms})$ close to the population median $\left(50^{\text {th }}\right.$ percentile $)$, while the bottom row shows corresponding images for a PA pregnancy at 235 days gestation with a median T2* (= 26 $\mathrm{ms})$ in the $1^{\text {st }}$ percentile. Depression of the placental $\mathrm{T} 2^{*}$ in the latter is clearly apparent in panel D.

Figure 3A shows the measured dependence of placental $\mathrm{T}^{*}$ across gestation in UN pregnancies. This quantity decreased continuously throughout pregnancy, beginning at a relatively high plateau level early in gestation, then dropping increasingly rapidly to an inflection point around 30 weeks before approaching a second, lower plateau in late gestation. Model fitting via nonlinear least squares regression to a logistic function is shown by the solid black curve, with 95\% fit confidence intervals $(\mathrm{Cl})$ indicated by the dashed lines and $95 \%$ fit prediction intervals $(\mathrm{PI})$ by the dot-dashed lines. Figure 3B plots the corresponding data and regression curves for SA (green) and PA (red) pregnancies, with the best fit and $95 \% \mathrm{Cl}$ curves from UN pregnancies shown in gray for reference. The model fit for the PA outcome group had significantly lower modeled T2* than UN pregnancies starting at 15 weeks and continuing through 33 weeks gestation, while the model fit for the SA outcome group was not significantly different from that for UN pregnancies at any point in gestation.

Site-dependent data and regressions for UN pregnancies are shown in Figure 3C for OHSU (blue) and Utah (red), with fit and $95 \% \mathrm{Cl}$ for all UN again plotted in gray. While the resulting curves are quite similar in shape, the Utah T2* data for UN pregnancies are consistently lower than the corresponding OHSU data, and the difference between the two is statistically significant between 15 and 29 weeks gestation. The observed site-specific differences in T2* in UN placentas can be accurately described with a simple model (see Methods) that characterizes these differences in terms of corresponding site differences in maternal hemoglobin and SpO2 levels, the known dependence of MRI signal on deoxyhemoglobin concentration, and a gestation-dependent maternal placental blood volume fraction $\left(v_{m p b}(t)\right)$ term that varies from approximately $15 \%$ early in gestation to approximately $35 \%$ by late gestation. Hemoglobin and $\mathrm{SpO} 2$ variation between imaging sites can be explained by the 
180

181

182

183

184

185

186

187

188

189

190

191

192

193

194

195

196

197

198

199

200

201

202

203

204

205

206

207

208

209

210

211

altitude difference, with the University of Utah at 4,840 feet above sea level while OHSU lies roughly 450 feet above sea level.

Median voxel-level relative measurement uncertainty in placental T2* data for UN pregnancies was $\pm 7.0 \%$, was comparable in both SA $( \pm 6.1 \%)$ and PA $( \pm 6.2 \%)$ pregnancies, and was significantly higher in the Utah studies than at OHSU $( \pm 5.8 \%$ for OHSU, $10.3 \%$ for Utah, $\mathrm{p}<0.001)$. In addition to stratifying based on pregnancy outcome and study site, the dependence of gestational T2* measurements in UN pregnancies on fetal sex, maternal age, and maternal body mass index (BMI) was evaluated (not plotted), with no significant differences among any of these. Excluding measurements not meeting the heuristic data quality criteria described in the methods did not significantly alter any reported results.

The average rate of change in placental T2* with gestation, computed from the centered finite difference of measurements in each individual pregnancy at successive time points, is plotted for UN pregnancies in Figure 3D, for PA (red), and SA (green) pregnancies in Figure 3E, and for OHSU (blue) vs. Utah (red) UN in Figure 3F. Model regressions to these data using the time derivative of the logistic function are displayed as in Figures $3 \mathrm{~A}-3 \mathrm{C}$. As with the $\mathrm{T} 2^{*}$ data themselves, the rate of change data for UN and SA pregnancies are not significantly different at any point during gestation. In contrast, the rate of change in PA pregnancies is nearly constant and shows a significantly larger rate of decrease in early and mid-gestation (up to 24 weeks) relative to UN. The rate of T2* decrease with gestation was found to be slightly, but significantly, larger in OHSU UN pregnancies than in Utah UN from 28 weeks gestation onward.

Receiver operating characteristic (ROC) curves for T2*

Histograms of T2* $z$-values for uncomplicated normal (blue), primary adverse outcome (red), and secondary abnormal outcome pregnancies (green) are plotted in Figure 4, where the uncomplicated pregnancies were used as the reference distribution. As expected, the distribution of z-scores for UN pregnancies is symmetrical and centered on zero (mean=0.0, $S D=1.0)$. Z-scores for the PA pregnancies are relatively symmetrical but broader and with a significant left shift (mean=-1.0, SD=1.49, $p<0.001$ ), while the distribution for SA pregnancies is shifted leftward (mean=-0.15, SD=1.34, $\mathrm{p}<0.001$ ) and notably skewed, suggesting the possibility of two subpopulations within these data.

The distributions of T2* percentiles derived from the z-score data are presented in bar chart form in Figure 5, with twenty equally-spaced bins spanning from 0 to 100 . The distribution of T2* percentiles in the UN population (blue) is, as expected, essentially uniform across the entire 
212 range, with roughly $5 \%$ of observations lying in each bin, while SA (green) pregnancies show

213 modest enrichment at low values. In contrast, the PA pregnancies lie primarily in the lowest (0-

$2145 \%$ ) bin, with nearly $35 \%$ of the adverse studies lying in that range and $44 \%$ in the lowest $10 \%$

215 of T2* measurements.

216 Figure 6 shows receiver operating characteristic (ROC) curves for the entire population across

217 gestation (leftmost column), and for data separated into early (10-20 weeks), mid (20-30

218 weeks), and late (30+ weeks) gestation (second through fourth columns). ROCs for both sites

219 are plotted in the top row, those computed using only OHSU data in the middle row, and those

220 computed using only the Utah data in the bottom row. For both sites across all gestational time

221 points, the area under the curve (AUC) or C-statistic for placental T2* and PA pregnancy

222 outcome is 0.71 , with mid-gestation showing the strongest predictive power (AUC of 0.76). C-

223 statistics are consistently higher in the OHSU cohort than the Utah cohort, with the strongest C-

224 statistic overall for OHSU studies in mid-gestation (AUC=0.82), and the weakest for Utah

225 studies in late-gestation ( $A \cup C=0.37$ ). The maximum in Youden's $\mathrm{J}$ statistic, $J_{\text {max }}$, is indicated by

226 the red stars on the ROC curves of Figure 6, and the corresponding optimal cutoff threshold in

227 T2* percentile relative to $\mathrm{UN}$ is indicated in the legends as $C_{\text {opt }}$.

$228 \quad$ Placental T1

229 Quantitative T1 values in UN pregnancies (acquired in OHSU participants only) showed linear

230 decrease with gestation at an average rate of -26.9 ms/week from approximately 2200 ms early

231 in gestation to roughly 1600 ms late in gestation. Neither SA nor PA pregnancies showed any

232 statistically significant differences in the evolution of T1 during pregnancy relative to UN,

233 suggesting that placental T1 is not a useful metric for characterization of placental dysfunction.

234 Maternal hemoglobin and oxygen saturation

235 Maternal hemoglobin level decreased linearly throughout gestation in UN pregnancies at an

236 average rate of $-0.046 \mathrm{mmol} /$ week and was significantly higher in the Utah cohort than the

237 OHSU cohort (mean difference $1.04 \pm 0.90 \mathrm{mmol}, \mathrm{p}<0.001$ ). Maternal hemoglobin was

238 significantly higher in the PA pregnancies compared to UN (mean difference $0.36 \pm 1.04 \mathrm{mmol}$,

$239 \mathrm{p}<0.001)$. There was no difference in hemoglobin between UN and SA pregnancies.

240 Maternal SpO2 in UN pregnancies was found to be essentially constant throughout gestation

241 (mean 97.0\%) but was significantly lower in the Utah cohort than the OHSU cohort (mean 
242 difference $-2.0 \pm 3.0 \%, p<0.001)$. Neither SA nor PA pregnancies were associated with

243 statistically different maternal $\mathrm{SpO} 2$ values or trends relative to UN.

244 Placental volume

245 Placental volume increased linearly during gestation at an average rate of $32.2 \mathrm{~cm}$ ^3/week

246 beginning between 11 and 12 weeks, with no significant difference between UN pregnancies at

247 the two sites. Our placental volume measurements are also highly consistent with those

248 reported by Leon et al. (46) in the overlapping gestational age range. Volume in SA pregnancies

249 was not significantly different than that of UN, while PA pregnancies showed a slightly lower rate

250 of growth $\left(30.0 \mathrm{~cm}^{\wedge} 3 /\right.$ week) leading to significantly lower volume from 20 weeks gestation

251 onward.

252 Regression modeling results

253 Model definitions, best fit parameter values, fit parameter uncertainties, and root-mean-square

254 (RMS) residual errors for regressions to all data and subsets discussed above are given in 255 Table 3.

\section{Discussion}

257 In this study, we characterize and model the sigmoidal evolution of T2* across gestation in 258 uncomplicated pregnancies and demonstrate that median placental T2* is markedly lower in 259 many pregnancies with adverse outcomes. We found lower values across gestation in 260 pregnancies with the primary adverse outcome and larger rate of decline in early and mid261 gestation when compared to uncomplicated normal pregnancies. Importantly, decreased 262 median T2* continues to correlate with adverse obstetric outcomes when quantified in both mid 263 and late gestation. The placenta is a dynamic organ which evolves over the entire course of 264 gestation, and possesses the capacity to adaptively develop in concert with the growing fetus.

265 As a result, it is not a fait accompli that poor placental function early in gestation persists 266 throughout pregnancy. However, our results suggest that, while T2* measurements acquired in 267 the mid-gestational time window (20-30 weeks) are most predictive of adverse pregnancy 268 outcomes, even data from the early gestational window (10-20 weeks) allow risk stratification.

269 The strong correlation of data observed between our two independent sites demonstrates that 270 this method is robust and has the potential to be transferable across different institutions.

271 Nevertheless, some relevant site-specific differences were observed that merit further

272 clarification. First, the birthweight in PA pregnancies among the Utah group was not 
273 significantly different from that of the UN pregnancies in that group, while the PA pregnancies in 274 the OHSU cohort had a significantly lower birthweight when compared to UN. This may simply 275 be accounted for by the higher proportion of SGA neonates in the OHSU cohort due to chance, 276 as population rates of adverse outcomes in these two groups are expected to be similar.

277 Second, the maternal Hgb was higher in Utah when compared to OHSU, which is expected 278 given the increased altitude in Salt Lake City, Utah when compared to Portland, Oregon. Third, 279 maternal oxygen saturation in the Utah patients was significantly lower than in the OHSU 280 population, also consistent with the physiologic impacts of altitude. In the UN population, it is 281 possible to entirely explain the observed differences between sites with a simple model 282 incorporating the site-specific hemoglobin and oxygen saturation differences along with a maternal placental blood volume term that varies across gestation, as described in the Methods.

284 The predictive power of T2* measurements for discriminating uncomplicated pregnancies from 285 primary adverse outcome pregnancies was much higher in the OHSU cohort than for Utah (AUC 0.80 vs 0.56). We suspect that this is due to site-specific differences in the prevalence of SGA and preeclampsia with severe features, both of which are relatively under-represented in the Utah group. Notably, in the Utah cohort, birthweights of neonates in the primary adverse outcome group were not statistically different than in uncomplicated pregnancies. Given that SGA and hypertensive diseases of pregnancy have multiple pathophysiologies with varying degrees of placental dysfunction, it is reasonable to propose that $\mathrm{T}^{*}$ quantification is primarily predictive of pathways linked to abnormalities attributable to perturbations of maternal placental blood flow and/or fetal oxygen uptake. It is possible that there is a secondary contribution due to the somewhat higher measurement error in the Utah data set as compared to OHSU, although the absolute measurement uncertainties are small for both study sites. Unfortunately, the modest number of PA pregnancies in our data set limits statistical power and precludes separation of the PA group into sub-categories.

The imaging methodology in this study is highly amenable to clinical translation. Placental MRI was performed using imaging protocols and pulse sequences that are available on virtually all modern MRI scanners, and analysis of these data requires only minimal post-processing to convert signal measurements to T2* values. Groundwork performed in our NHP models was key to both validation and translation of this methodology to human subjects by validating T2* mapping as a functional measure of maternal placental perfusion with confirmation by DCE-MRI $(33,34)$. While DCE-MRI is the gold-standard method for quantifying tissue perfusion via MRI, and despite the fact that we have demonstrated minimal placental permeability to passage of 
gadolinium-based contrast agents (GBCA) following in utero maternal administration (47, 48), a GBCA-free alternative alleviates potential reservations to use of MRI as a clinical diagnostic imaging tool for assessing placental health. In addition, because placental T2* is sensitive to the balance between oxygenated maternal blood delivery and fetal oxygen demand, it is particularly well-suited to identify problems stemming from inadequate placental oxygenation.

\section{Study strengths and limitations}

Our study has a number of strengths. It is the largest prospective study of placental MRI, and the most extensive study of $\mathrm{T}^{*}$, in particular. In addition, the longitudinal design enabled us to characterize the nonlinear evolution of T2* across pregnancy and provide reference values for both $\mathrm{T}^{*}$ itself and rate of change in $\mathrm{T} 2^{*}$ as gestation progresses. While studies of changes in T2*-weighted BOLD-EPI measurements in response to hyperoxygenation have a number of advantages, particularly in data acquisition efficiency and sensitivity to motion, they are generally semi-quantitative, introduce methodological complexity, and potentially perturb maternal and fetal hemodynamics and alter the physiologic mechanisms that determine normal oxygen transport across a gradient (49-51). In contrast, quantitative measurements of T2* are reflective of the balance between maternal delivery of oxygen and fetal demand, are reproducible across sites, and do not need ancillary experimental perturbations. The primary adverse composite outcome metric we developed was defined prior to, and independent of, MRI data analysis. Designation of pregnancy outcome was blinded to MRI data analysis and was conducted by four Maternal-Fetal Medicine physicians independently, increasing the rigor of our outcome designation. Similarly, to further reduce the potential for bias, MRI data processing was blinded to pregnancy outcome and was conducted independently prior to statistical analysis for association with adverse pregnancy outcomes. By utilizing common, commercially available MRI acquisition protocols, the work described here should be easy to reproduce at other institutions, facilitating its potential use both in future clinical studies and in clinical practice.

There are also a number of limitations to this study. Although it is the largest longitudinal MRI study in pregnancy performed to date, the number of adverse outcomes was small. This necessitated the utilization of a composite outcome, as is typical for obstetric studies. Our study population is relatively ethnically and racially homogeneous, so the conclusions we draw here may not be applicable to other populations. MRI was performed using 3 Tesla scanning hardware to increase sensitivity to changes in $\mathrm{T} 2^{*}$, but these systems are not currently the standard in obstetric imaging and are not as widely available as 1.5 Tesla systems. While we used consistent criteria encompassing many common complications, there is no gold standard 
definition of placental dysfunction, and our outcomes are heterogenous by nature. In particular, we have previously identified circumstances where pathology related to villous inflammation or malformation can cause elevated T2* (35) in the setting of adequate supply of maternal arterial blood to the placenta in conjunction with impaired trans-villous oxygen permeability, which could constitute a confounding factor in some pregnancies. As a result, further refinement may be required to detect abnormally high, as well as abnormally low $\mathrm{T} 2^{*}$, to accurately capture different types of placental pathology.

\section{Conclusion}

We present the results of a prospective longitudinal human study that demonstrate the potential of quantitative $\mathrm{T}^{*}$ mapping during pregnancy to identify increased risk for adverse obstetric outcome due to placental dysfunction, particularly in the setting of fetal growth restriction. Quantitative measures of placental T2* identified pregnancies at increased risk for adverse outcomes across all gestational ages in this study despite site-specific differences in maternal and neonatal demographics at the two institutions. Low median placental $\mathrm{T} 2^{*}$ was strongly correlated with low fetal birthweight, suggesting that the diagnostic utility of placental MRI may be enhanced by focusing on a specific adverse obstetric outcome, such as fetal growth restriction, rather than a composite adverse outcome. Improved diagnostics to identify pregnancies at risk of adverse outcomes may facilitate discovery of novel biomarkers, improved stratification of patients in clinical studies, and may allow for earlier modification of clinical management plans.

\section{Methods}

All protocols described in the following were approved by the Institutional Review Boards (IRB) at Oregon Health \& Science University (OHSU) and University of Utah Health Sciences Center (UUHSC), and study oversight was provided by an independent data and safety monitoring board.

\section{Study Design}

A longitudinal prospective cohort study of 316 pregnant women at two sites, both academic tertiary care centers with Level VI neonatal intensive care units: OHSU and the UUHSC for three MRI studies at the following gestational ages: 12-16 weeks, 26-28 weeks, and 32-34 weeks (ClinicalTrials.gov: NCT02749851). The original prospective observational study design 
had planned enrollment for 300 subjects to undergo the MRI studies in the aforementioned gestational windows. The rationale for the original exploratory study design was to facilitate characterization of $\mathrm{T} 2^{*}$ longitudinally during pregnancy and to minimize sensitivity to population variability in $\mathrm{T} 2^{*}$ values as a function of gestational age. A planned interim analysis in year 3 demonstrated tight correlation of $\mathrm{T}^{*}$ across study sites and within gestational age timepoints. As a result, the decision was made to open the gestational time windows for recruitment by $+/-8$ weeks to facilitiate improved characterization of the evolution of placental T2* throughout pregnancy. The larger recruitment windows impacted the number of MRIs per study participant as some participants recruited later in gestation were not be able to complete additional MRI studies depending on the gestational age at enrollment. The decreased frequency of repeat MRI per study subject however did facilitate recruitment and enrollment of a larger study cohort than originally planned.

Participants were recruited from the OHSU and UUHSC clinics where written informed consent was obtained with IRB approval. Pregnant women were recruited based on inclusion criteria for two subject groups: a low-risk cohort not at increased risk for adverse obstetric outcome and a high-risk group at increased risk for adverse outcomes based on prior clinical history. A third group of pregnant tobacco smokers was originally planned as a separate cohort but recruitment was abandoned due to lack of success in identification and enrollment.

Inclusion criteria

Inclusion criteria for both groups included pregnancy (defined by positive pregnancy test and certain menstrual history, or early ultrasound) identified prior to 16 weeks gestation, maternal age over 18 years of age, and ability to give informed consent. The inclusion criteria for the lowrisk cohort were all of the following: 1) no history of a second or third trimester pregnancy loss, 2) no history of fetal growth restriction, and 3) nonsmoker. The inclusion criteria for the high-risk group were one or more of the following: 1) history of pregnancy complicated by placental insufficiency in a previous singleton pregnancy defined by preeclampsia with severe features requiring preterm delivery, or preterm delivery due to placental insufficiency (fetal growth restriction, oligohydramnios, abnormal umbilical artery Doppler), or fetal growth restriction with neonatal weight $<10^{\text {th }}$ percentile delivered at term, or stillbirth attributed to placental cause, regardless of gestational age, 2) pregnancy at risk for placental insufficiency due to clinical comorbidities (i.e. chronic hypertension), or 3) history of spontaneous preterm birth $<34$ weeks.

\section{Exclusion criteria}


402 Exclusion criteria were maternal intellectual disability or incarceration, pregnancy with major

403 fetal anomalies known to be associated with abnormal fetal growth, active alcohol use during 404 pregnancy, medical conditions requiring ongoing treatment during pregnancy including cancer, 405 acute liver disease, chronic pulmonary disease requiring regular use of medication, history of 406 claustrophobia, metal implants, or other contraindication for MRI, and increased risk of 407 aneuploidy based on ultrasound findings and/or genetic testing.

408 Participant enrollment

409 Potential participants were identified through multiple modalities. The research team utilized 410 social media, which entailed Facebook advertisements and promotions via institutional

411 websites. The research teams also attended multiple pregnancy groups, such as prenatal group

412 intake meetings and events for pregnant women, as well as a Portland-based website for new

413 and expecting parents with resources and events. Potential research subjects were screened in

414 the OHSU electronic medical record system, and Utah appointment logs through a waiver of

415 authorization. Once participants were found via electronic medical records, they were

416 approached at their next prenatal appointment or sent a MyChart message with pertinent

417 research study information. When a potential subject reached out to the team via phone or

418 email with interest, a phone screening was conducted. The phone screening reviewed basic

419 eligibility inclusion and exclusion criteria, contact information, and additional preferences. If the

420 subject was found to be eligible, they would be scheduled for a visit in accordance with the 421 study protocol where they will start the visit with a detailed explanation of the study and followed

422 with the signature of the informed consent. If not eligible for the study or no longer interested,

423 they would be thanked for their time and interest.

424 Pregnancy outcome designation

425 Pregnancies were categorized postnatally into three groups: a) uncomplicated normal 426 pregnancies (UN), b) primary adverse outcome pregnancies (PA), and c) secondary abnormal 427 pregnancies (SA). Uncomplicated pregnancies were defined as those with term (37 weeks or 428 beyond) delivery with birthweight between the $5^{\text {th }}$ and $95^{\text {th }}$ percentile, without gestational 429 hypertensive disease, and not meeting any criteria for the primary adverse outcome or 430 secondary abnormal outcome.

431 The primary adverse outcome group was defined using a composite including hypertensive 432 disorders of pregnancy, with birthweight below the $5^{\text {th }}$ percentile by Oken (43), and stillbirth or 433 fetal death. Hypertensive disorders of pregnancy included gestational hypertension, 
preeclampsia without severe features, preeclampsia with severe features, Hemolysis, elevated liver enzymes, and low platelet count (HELLP) syndrome, and eclampsia. These were defined by ACOG criteria (52). Gestational hypertension was defined as systolic blood pressure of 140 $\mathrm{mm} \mathrm{Hg}$ or more or a diastolic blood pressure of $90 \mathrm{~mm} \mathrm{Hg}$ or more, or both, on two occasionas at least 4 hours apart at or beyond 20 weeks of gestation in someone without chronic hypertension. Preeclampsia was defined as elevated blood pressure (as in gestational hypertension) plus proteinuria (300mg or more in a 24 hour urine collection or urine protein/creatinine ratio of 0.3 or more) or presence of severe features without proteinuria. Severe features included systolic blood pressure of $160 \mathrm{~mm} \mathrm{Hg}$ or more or diastolic blood pressure of $110 \mathrm{~mm} \mathrm{Hg}$ or more on two occasions at least 4 hours apart, thrombocytopenia with platelet count $<100 \times 10^{9} / \mathrm{L}$, liver enzymes more than twice the upper limit of normal, severe persistent right upper quadrant pain or epigastric pain, serum creatinine $>1.1 \mathrm{mg} / \mathrm{dL}$, pulmonary edema, new headache unresponsive to medication, and/or visual disturbances. HELLP syndrome is defined by thromboycytopenia with platelet count $<100 \times 10^{9} / \mathrm{L}$, liver enzymes more than twice the upper limit of normal, and evidence of hemolysis (LDH > 600 IU/L). Eclampsia was defined as seizure in absence of alternative etiology and with concomitant criteria for preeclampsia.

The secondary abnormal outcome group included pregnancies complicated by maternal chronic hypertension without superimposed preeclampsia, fetal genetic and/or anatomic anomalies; spontaneous preterm birth due to preterm labor, cervical insufficiency, and/or preterm premature rupture of the membranes (PPROM); placental abruption, chorioamnionitis, and/or birthweight greater than the $95^{\text {th }}$ percentile by Oken (43). Our goal in creating this group was to capture pregnancy morbidity that is typically due to non-placental causes. Though morbidity can be significant in this group, our hypothesis was that our MRI protocol would not show strong association with these outcomes.

The primary adverse clinical composite outcome was developed pragmatically. Gestational hypertensive disease, preeclampsia, low birth weight, and fetal death/stillbirth are all linked to placental dysfunction $(3,50,51)$. We acknowledge that this is not a pure phenotype, and there are multiple pathways to each of these outcomes, let alone the composite. However, this composite is clinically meaningful when attempting to capture morbidity and mortality due to placental insufficiency and there is significant overlap between the individual outcomes. Thus, in order to assess utility of MRI as a tool for either prediction or targeting of therapeutics, assessing these outcomes in toto is appropriate. 
Adjudication of the primary adverse outcomes and secondary abnormal outcomes was performed independently by both of the Maternal-Fetal Medicine physicians at each site (OHSU: KJG, AEF; Utah: JMP, NRB). Any discordance between assessment of outcome was then discussed and reconciled prior to final determination. The authors determining the outcomes were blind to the MRI data and analysis. Similarly, MRI data acquisition and quality control was performed blind to pregnancy outcome group.

\section{Magnetic Resonance Imaging}

Maternal blood draws were performed prior to each scan and hemoglobin level measured using iStat (Abbott, Princeton, NJ) and/or fingerstick. Pulse oximetry (Zacurate 500BL) was used to determine maternal blood oxygen saturation level before each MRI study.

$\mathrm{MRI}$ in pregnant human volunteers was performed at up to three consecutive time points during pregnancy, ranging from 11 to 38 weeks gestation, on 3T Siemens Prisma scanner hardware using vendor spine and body array coils. Following localization of the uterus and placenta and acquisition of T2-HASTE anatomic imaging in three planes (axial, coronal, and sagittal), breathhold multi-slice multi-echo gradient echo (MEGE) images were acquired in an axial orientation for T2* mapping, spanning the entire uterus with a spatial resolution of $1.75 \times 1.75 \times 3.5 \mathrm{~mm}$, at six in-phase echo times (TE): 4.92/9.84/19.68/29.52/36.90/49.20 ms with a repetition time (TR) of $116 \mathrm{~ms}$. Number of slices and in-plane coverage were adjusted as necessary to achieve complete coverage of the uterus and avoid image wrap. Breath hold duration was maintained below 10 seconds per acquisition to minimize patient discomfort. In OHSU studies, 3D variable flip angle T1 mapping was also performed with full placental coverage, using the Siemens Maplt protocol with flip angles of 3 and 15 degrees, including B1 correction (resolution 0.9x0.9x4 mm, $\mathrm{TR}=5.01, \mathrm{TE}=2.23 \mathrm{~ms}$ ).

\section{Image and data analysis}

All post-processing, data modeling, and statistical analysis was performed in MATLAB (Mathworks, Natick MA) using standard library functions where available and custom in-house software otherwise. Quantitative T2* values were determined by weighted log-linear regression of MEGE signal magnitude measurements as described in detail in Schabel et al (34). Voxellevel uncertainty in estimated $\mathrm{T} 2{ }^{*}$ values was computed from regression covariance matrices and propagation of errors analysis using measured magnitude noise levels in source MRI data. Three dimensional placental regions-of-interest (ROIs) were hand drawn on T2* maps, using co-registered T2-HASTE images as an anatomic reference when the placental-myometrial 
and/or placental-fetal boundaries were indistinct. Slices with excessive image quality degradation arising from maternal and/or fetal motion were excluded from analysis. Readers were blinded to patient status and pregnancy outcome. Binary masks were derived from the placental ROls, with T2* values of 250 ms or more being excluded from further analysis; such large values are associated with signal contamination by amniotic fluid. Placental volume was computed by summing the number of voxels in each slice of this binary mask multiplied by the per-voxel volume. Where slices were missing due to motion, volumes were estimated from adjacent slices using linear interpolation. In the OHSU cohort, median placental T1 was determined by spatially resampling measured T1 maps onto the T2* image volumes and applying placental ROIs.

Histogram analysis was used to compute median placental T2* for each study. Median relative fit uncertainty in $\mathrm{T}^{*}$ (the ratio of the model estimated sigma-T2* to estimated T2*) was computed as a measure of measurement quality. A heuristic quality statistic was determined by computing the fraction of placental voxels for which the relative fit uncertainty was $<=0.25$.

Sensitivity of the data analysis pipeline to data quality was evaluated by re-running the entire statistical post-processing pipeline for both the entire set of studies and for a reduced set where studies with either median relative uncertainty in the highest $10 \%$ or heuristic quality in the lowest $10 \%$ were excluded. Potential bias stemming from variation in the number of scans per patient ranging from one to three was considered by re-running the post-processing pipeline with scan weight distributed evenly per patient, rather than per scan.

Trends in maternal SpO2, maternal hemoglobin concentration, placental volume, and placental T1 across gestational age were found to be well fit with a linear model. Gestational trends in T2* were modeled sequentially with linear and quadratic polynomials as well as with a logistic function $\left(T_{2}^{*}(t)=p_{1} /\left(1+\exp \left(p_{2}\left(t-p_{3}\right)\right)\right)+p_{4}\right)$, which was chosen based on the sigmoidal behavior observed in median T2* curves in normal pregnancies. Regression modeling used polyfit for polynomial fitting and nlinfit for nonlinear model fitting to $\mathrm{T}^{*}$ data, with regressions weighted by measurement uncertainties. The Bayes Information Criterion (BIC) was used to account for different numbers of free parameters for model selection. Based on the BIC results, the sigmoid function was determined to have the best fit to measured T2* data. Intraindividual time derivatives in $\mathrm{T}^{*}$ across gestational age were approximated as pairwise centered finite differences from measurements acquired at sequential gestational time points: $\left(\Delta T_{2}^{*} / \Delta t\right)\left(\left(t_{1}+t_{2}\right) / 2\right)=\left(T_{2}^{*}\left(t_{2}\right)-T_{2}^{*}\left(t_{1}\right)\right) /\left(t_{2}-t_{1}\right)$. The resulting data were fit with the time derivative of the logistic function: $\left(\Delta T_{2}^{*} / \Delta t\right)(t)=-p_{1} p_{2} \exp \left(p_{2}\left(t-p_{3}\right)\right) /\left(1+\exp \left(p_{2}\left(t-p_{3}\right)\right)\right)^{2}$. 
532 Fit covariance matrices were used to compute model parameter uncertainties as well as $95 \%$

533 confidence and prediction intervals for all regressions. Model regressions were considered to be

534 significantly different where their $95 \%$ confidence intervals were non-overlapping. In addition to

535 the three primary study groups (UN pregnancies, SA pregnancies, and PA pregnancies),

536 differences between UN at the two study sites were compared, along with differences based on

537 fetal sex, maternal age, and maternal BMI.

538 A model describing the observed inter-site T2* differences across gestation between OHSU and

539 Utah sub-populations was developed starting from the assumption that these differences arise

540 from corresponding site-dependent differences in maternal hemoglobin and SpO2 levels, along

541 with a maternal placental blood volume fraction that evolves through pregnancy. The resultant

542 shift is most easily described in terms of differences in the transverse relaxation rate, defined as

$543 \quad R_{2}^{*}=1 / T_{2}^{*}$, between site A (OHSU) and site B (Utah) using the known transverse relaxivity of

544 deoxyhemoglobin $\left(r_{2}^{*}=20.2 / \mathrm{mmol} / \mathrm{s}\right)$ and blood deoxyhemoglobin concentrations computed from

545 measured maternal SpO2, along with the maternal placental blood volume fraction $\left(v_{m p b}\right)$ :

546

$$
\Delta R_{2}^{*}(t)=r_{2}^{*}\left([H b(t)]_{A}\left(100-\left(S_{p} O_{2}(t)\right)_{A}\right)-[H b(t)]_{B}\left(100-\left(S_{p} O_{2}(t)\right)_{B}\right)\right) v_{m p b}(t)
$$

547 Combining this expression with the modeled dependence of maternal SpO2 and hemoglobin

548 levels for the two sites allows us to solve for the gestation-dependent value of $v_{m p b}(t)$ that

549 corresponds to observed differences in placental T2*. This parameter is a volume fraction that

550 should correspond approximately to in vivo intervillous volume (55), and is physically-

551 constrained to lie in the closed interval $[0,1]$.

552 Z-scores were computed for all placental T2* measurements using the model regression and

553 prediction intervals for UN pregnancies, and T2* percentiles were calculated from the

554 cumulative distribution function (CDF) of the corresponding normal distribution. Receiver

555 operating characteristic (ROC) curves were then generated from the T2* percentiles for all

556 individual studies in UN vs. PA pregnancies (SA pregnancies were omitted from this analysis).

557 Studies were also grouped into three gestational time windows: early gestation (10-20 weeks),

558 mid-gestation (20-30 weeks), and late gestation (30-40 weeks) in order to assess the

559 performance of this metric across gestation, and ROCs were separately computed for OHSU

560 and Utah data to compare the site-specific performance.

561 Statistics 
562 All p-values for continuous variables presented in this manuscript were computed using a two563 tailed Kolmogorov-Smirnov test. The chi-square proportion test was used to compute p-values 564 of binary variables. Significance of differences in model regressions was assessed using the 565 presence or absence of overlapping 95\% confidence intervals.

566

567 Tables

568 Table 1: Demographic data from the study populations at OHSU and Utah.

569 Table 2: Breakdown of prenatal conditions in the primary adverse outcome group for entire PA 570 population and by site.

571 Table 3: Regression models, best fit parameter values and estimated parameter uncertainties, 572 and RMS fit residual values for model fits of gestational trends in $T 2^{*}, \Delta \mathrm{T} 2^{*} / \Delta \mathrm{GW}$, maternal 573 hemoglobin, maternal blood oxygen saturation, in vivo placental volume, and T1. Fits are 574 presented for the aggregate data set along with separate regressions to the OHSU and Utah 575 subpopulations for normal pregnancies.

576

577 


\section{Acknowledgements}

579 This work was supported by: NICHD Human Placenta Project U01 HD 087182 (Frias) and R01

580 HD 086331 (Frias): NIH S10OD021701 for the 3T Siemens Prisma MRI instrument, housed in

581 OHSU's Advanced Imaging Research Center: NIH S100D018224 for the High Performance

582 Computing Cluster, housed in OHSU's Advanced Imaging Research Center: the Oregon Clinical

583 \& Translational Research Institute grant which supported the use of REDCap (Research

584 Electronic Data Capture) for data abstraction (CTSA Award No.: UL1TR002369).

585

586

Author contributions.

587 MCS, VHJR, CDK, RMS, AEF: Conceived and designed study. AEF, VHJR, MR, KJG, MWV, $588 \mathrm{KS}$ : Coordinated and facilitated subject recruitment, enrollment and participation in the study. 589 MCS, VHJR, KJG, AEF, NRB, JMP, CDK, BP, MWV, RMS: Reviewed the data and aided in 590 experimental design. MCS, VHJR, KJG, AEF: drafted the manuscript. All authors reviewed the 591 manuscript. MCS: Supervised and acquired MRI data, developed software for MRI data post592 processing and analysis, performed MRI data analysis. ADS, AMW, JOL: Assisted with MRI 593 data analysis. MR, JEG, KS, KH: Assisted and facilitated RedCap data entry. MCS, BP:

594 Designed and performed statistical analysis.

595

596 
598 Figure 1: Enrollment flow chart.

599 A flow chart detailing numbers of prospective patients screened, consented, and enrolled at 600 both study sites, along with numbers of completed MRI studies meeting quality criteria for 601 inclusion in data analysis presented here.

602 Figure 2: Comparison of anatomic imaging (T2-weighted HASTE, left column) and placental 603 T2* mapping (right column) in a uncomplicated normal pregnancy at 232 days gestation (top 604 row, panels A \& B) with those from a primary adverse pregnancy at 235 days gestation 605 presenting with severe preeclampsia (bottom row, panels $C \& D$ ). The placenta is indicated by 606 the dashed blue outlines overlaid on the T2* maps.

607 Figure 3: Gestational dependence of placental T2* values.

608 Median T2* values for each completed study, computed over the entire placenta, are plotted as 609 a function of gestational age at time of imaging in the three panels in the left column (panels A, $610 \mathrm{~B}, \mathrm{C}$ ), while corresponding rates of change in placental T2* between repeated imaging time 611 points within the same individual are plotted as a function of gestational age in the right column

612 (panels D, E, F). The upper row plots these quantities for normal pregnancies, the middle row 613 for abnormal (green) and adverse (red) pregnancies, and the bottom row for normal 614 pregnancies stratified by site (OHSU in blue, Utah in red). In all graphs, model regression 615 curves (using the functions defined in Table 2) are indicated by the thick solid lines, the $95 \%$ 616 confidence intervals by the dashed lines, and the 95\% prediction intervals by the dot-dashed 617 lines. The best fit and $95 \% \mathrm{Cl}$ curves from the plots in the upper row are shown in gray in the 618 middle and bottom rows for reference.

619 Figure 4: Histograms of T2* Z-scores in normal, abnormal, and adverse pregnancies.

620 Z-score histograms shown are computed using prediction intervals for sigmoid model regression

621 to T2* measurements in UN pregnancies, applied to individual studies in UN (blue), PA (red), 622 and SA (green) pregnancies.

623 Figure 5: Bar chart of distribution of measured T2* percentiles for uncomplicated normal, 624 primary adverse, and secondary abnormal pregnancies.

625 Figure 6: Receiver operator characteristic (ROC) curves for T2* measurements in pregnancies 626 with our primary adverse outcome relative to uncomplicated normal pregnancies. 
627 The points where Youden's $J$ is maximized are indicated by the stars. Area under the curve (AUC), $J_{\max }$, 628 and the corresponding optimal cutoff threshold in T2* percentile relative to UN $\left(C_{\text {opt }}\right)$ are given in the 629 figure legend for each panel. 
Table 1: Demographic data for uncomplicated (UN), primary adverse (PA), and secondary abnormal (SA) groups, for entire study population and 631 separated by study site.

\begin{tabular}{|c|c|c|c|c|c|c|c|c|c|}
\hline & UN & & & PA & & & SA & & \\
\hline & All & OHSU & Utah & All & $\mathrm{OHSU}$ & Utah & All & $\mathrm{OHSU}$ & Utah \\
\hline $\begin{array}{l}\text { Maternal age at } \\
\text { conception }\end{array}$ & $31.2(4.6)$ & $31.8(5.1)$ & $30.4(4.0)$ & $31.7(5.1)$ & $32.2(5.3)$ & $31.0(4.9)$ & $31.0(5.4)$ & $30.9(5.7)$ & $31.4(4.7)$ \\
\hline \multicolumn{10}{|l|}{ Race } \\
\hline White & 175 (81.4\%) & 97 (79.5\%) & 78 (83.9\%) & $55(75.3 \%)$ & 29 (69.0\%) & 26 (83.9\%) & $42(73.7 \%)$ & 27 (69.2\%) & 15 (83.3\%) \\
\hline African Descent & $9(4.2 \%)$ & $9(7.4 \%)$ & $0(0.0 \%)$ & $1(1.4 \%)$ & $1(2.4 \%)$ & $0(0.0 \%)$ & $2(3.5 \%)$ & $1(2.6 \%)$ & $1(5.6 \%)$ \\
\hline Native American & $5(2.3 \%)$ & $3(2.5 \%)$ & $2(2.2 \%)$ & $0(0.0 \%)$ & $0(0.0 \%)$ & $0(0.0 \%)$ & $3(5.3 \%)$ & $2(5.1 \%)$ & $1(5.6 \%)$ \\
\hline Asian Indian & $4(1.9 \%)$ & $3(2.5 \%)$ & $1(1.1 \%)$ & $1(1.4 \%)$ & $0(0.0 \%)$ & $1(3.2 \%)$ & $0(0.0 \%)$ & $0(0.0 \%)$ & $0(0.0 \%)$ \\
\hline Other Asian & $10(4.7 \%)$ & $6(4.9 \%)$ & $4(4.3 \%)$ & $6(8.2 \%)$ & $4(9.5 \%)$ & $2(6.5 \%)$ & $2(3.5 \%)$ & $2(5.1 \%)$ & $0(0.0 \%)$ \\
\hline Native Hawaiian & $0(0.0 \%)$ & $0(0.0 \%)$ & $0(0.0 \%)$ & $0(0.0 \%)$ & $0(0.0 \%)$ & $0(0.0 \%)$ & $1(1.8 \%)$ & $1(2.6 \%)$ & $0(0.0 \%)$ \\
\hline Pacific Islander & $0(0.0 \%)$ & $0(0.0 \%)$ & $0(0.0 \%)$ & $1(1.4 \%)$ & $0(0.0 \%)$ & $1(3.2 \%)$ & $1(1.8 \%)$ & $1(2.6 \%)$ & $0(0.0 \%)$ \\
\hline Other & $1(0.5 \%)$ & $1(0.8 \%)$ & $0(0.0 \%)$ & $0(0.0 \%)$ & $0(0.0 \%)$ & $0(0.0 \%)$ & $0(0.0 \%)$ & $0(0.0 \%)$ & $0(0.0 \%)$ \\
\hline Unknown & $1(0.5 \%)$ & $1(0.8 \%)$ & $0(0.0 \%)$ & $0(0.0 \%)$ & $0(0.0 \%)$ & $0(0.0 \%)$ & $0(0.0 \%)$ & $0(0.0 \%)$ & $0(0.0 \%)$ \\
\hline Hispanic & $10(4.7 \%)$ & $2(1.6 \%)$ & $8(8.6 \%)$ & $9(12.3 \%)$ & $8(19.0 \%)$ & $1(3.2 \%)$ & $6(10.5 \%)$ & $5(12.8 \%)$ & $1(5.6 \%)$ \\
\hline $\begin{array}{l}\text { Pre-pregnancy } \\
\text { BMI }\end{array}$ & $24.4(4.4)$ & $24.7(4.8)$ & $24.0(3.9)$ & $26.3(5.9)$ & $27.4(6.5)$ & $24.8(4.8)$ & $36.7(5.8)$ & $27.2(6.2)$ & $25.4(4.6)$ \\
\hline
\end{tabular}

632

633 
Table 2: Breakdown of prenatal conditions in the primary adverse outcome group for entire PA population and by site.

\begin{tabular}{l|c|c|c|c|}
\hline Outcome & $\begin{array}{c}\text { Total } \\
\mathbf{N}=74\end{array}$ & $\begin{array}{c}\text { OHSU } \\
\mathbf{N}=39\end{array}$ & $\begin{array}{c}\text { Utah } \\
\mathbf{N}=31\end{array}$ & p-value \\
\hline PIH & $59(84.3 \%)$ & $34(87.2 \%)$ & $25(80.6 \%)$ & 0.68 \\
\hline Gestational HTN & $22(31.4 \%)$ & $13(33.3 \%)$ & $9(29.0 \%)$ & 0.90 \\
\hline Pre-e w/o sev & $9(12.8 \%)$ & $3(7.7 \%)$ & $6(19.4 \%)$ & 0.28 \\
\hline Pre-e w/sev & $28(40.0 \%)$ & $18(46.2 \%)$ & $10(32.2 \%)$ & 0.35 \\
\hline SGA & $14(20.0 \%)$ & $9(23.1 \%)$ & $4(16.1 \%)$ & 0.44 \\
\hline Stillbirth or fetal loss & $4(5.7 \%)$ & $1(2.6 \%)$ & $3(9.7 \%)$ & 0.45 \\
\hline Placental abruption & $2(2.8 \%)$ & $0(0.0 \%)$ & $2(6.4 \%)$ & 0.38 \\
\hline Both PIH + SGA & $5(7.1 \%)$ & $3(7.7 \%)$ & $2(6.4 \%)$ & 0.79 \\
\hline Preterm birth (<37w) & $31(35.7 \%)$ & $13(33.3 \%)$ & $12(38.7 \%)$ & 0.83 \\
\hline
\end{tabular}




\begin{tabular}{|c|c|c|c|c|c|}
\hline & $p_{1}$ & $p_{2}$ & $p_{3}$ & $p_{4}$ & $R M S_{\text {fit }}$ \\
\hline Median T2* (ms) & \multicolumn{4}{|c|}{$T_{2}^{*}(t)=p_{1} /\left(1+\exp \left(p_{2}\left(t-p_{3}\right)\right)\right)+p_{4}$} & \\
\hline UN pregnancies & $-59.2(+/-6.5)$ & $-0.24(+/-0.04)$ & $29.3(+/-0.8)$ & $84.6(+/-1.3)$ & $+/-9.6 \mathrm{~ms}$ \\
\hline OHSU only & $-59.3(+/-7.6)$ & $-0.26(+/-0.05)$ & $29.4(+/-0.9)$ & $86.3(+/-1.5)$ & $+/-9.5 \mathrm{~ms}$ \\
\hline Utah only & $-62.8(+/-13.9)$ & $-0.20(+/-0.06)$ & $29.5(+/-1.8)$ & $83.6(+/-2.8)$ & $+/-9.2 \mathrm{~ms}$ \\
\hline SA pregnancies & $-53.7(+/-11.3)$ & $-0.28(+/-0.11)$ & $26.8(+/-1.3)$ & $85.4(+/-4.0)$ & $+/-12.5 \mathrm{~ms}$ \\
\hline PA pregnancies & $-57.6(+/-22.9)$ & $-0.19(+/-0.11)$ & $25.2(+/-2.0)$ & $82.9(+/-10.7)$ & $+/-13.8 \mathrm{~ms}$ \\
\hline$\Delta \mathrm{T} 2 * / \Delta \mathrm{GW}(\mathrm{ms} /$ week) & \multicolumn{5}{|c|}{$\Delta T_{2}^{*} / \Delta t(t)=-p_{1} p_{2} \exp \left(p_{2}\left(t-p_{3}\right)\right) /\left(1+\exp \left(p_{2}\left(t-p_{3}\right)\right)\right)^{2}$} \\
\hline UN pregnancies & $-64.9(+/-4.2)$ & $-0.20(+/-0.01)$ & $30.1(+/-0.6)$ & & $+/-1.2 \mathrm{~ms} / \mathrm{wk}$ \\
\hline OHSU only & $-75.5(+/-9.5)$ & $-0.19(+/-0.02)$ & $31.3(+/-1.1)$ & & $+/-1.0 \mathrm{~ms} / \mathrm{wk}$ \\
\hline Utah only & $-60.2(+/-5.0)$ & $-0.20(+/-0.02)$ & $29.6(+/-0.7)$ & & $+/-1.1 \mathrm{~ms} / \mathrm{wk}$ \\
\hline SA pregnancies & $-79.0(+/-22.0)$ & $-0.16(+/-0.04)$ & $30.7(+/-2.6)$ & & $+/-1.4 \mathrm{~ms} / \mathrm{wk}$ \\
\hline PA pregnancies & $-5574.6(+/-1 \mathrm{e} 6)$ & $-0.02(+/-0.24)$ & $225.9(+/-2 e 5)$ & & $+/-1.4 \mathrm{~ms} / \mathrm{wk}$ \\
\hline$[\mathrm{Hb}](\mathrm{mg} / \mathrm{dl})$ & \multicolumn{4}{|c|}{$[H b](t)=p_{1}+p_{2} t$} & \\
\hline UN pregnancies & $13.27(+/-0.15)$ & $-0.046(+/-0.006)$ & & & $+/-1.02 \mathrm{mg} / \mathrm{dl}$ \\
\hline OHSU only & $12.86(+/-0.17)$ & $-0.047(+/-0.007)$ & & & $+/-0.86 \mathrm{mg} / \mathrm{dl}$ \\
\hline Utah only & $13.78(+/-0.21)$ & $-0.042(+/-0.008)$ & & & $+/-0.90 \mathrm{mg} / \mathrm{dl}$ \\
\hline SA pregnancies & $12.65(+/-0.32)$ & $-0.018(+/-0.013)$ & & & $+/-0.92 \mathrm{mg} / \mathrm{dl}$ \\
\hline PA pregnancies & $13.46(+/-0.31)$ & $-0.039(+/-0.012)$ & & & $+/-1.04 \mathrm{mg} / \mathrm{dl}$ \\
\hline SpO2 (\%) & \multicolumn{4}{|c|}{$S p O_{2}(t)=p_{1}+p_{2} t$} & \\
\hline UN pregnancies & $97.05(+/-0.57)$ & $0.003(+/-0.021)$ & & & $+/-2.4 \%$ \\
\hline OHSU only & $98.37(+/-0.38)$ & $-0.010(+/-0.014)$ & & & $+/-1.2 \%$ \\
\hline Utah only & $95.24(+/-1.03)$ & $0.031(+/-0.037)$ & & & $+/-3.0 \%$ \\
\hline SA pregnancies & $98.54(+/-0.65)$ & $-0.043(+/-0.025)$ & & & $+/-1.4 \%$ \\
\hline PA pregnancies & $95.99(+/-1.16)$ & $0.052(+/-0.044)$ & & & $+/-3.1 \%$ \\
\hline $\begin{array}{l}\text { Placental volume } \\
\text { (cm^3) }\end{array}$ & \multicolumn{4}{|c|}{$V(t)=p_{1}+p_{2} t$} & \\
\hline UN pregnancies & $-372.4(+/-17.6)$ & $32.2(+/-0.68)$ & & & $+/-122 \mathrm{~cm}^{\wedge} 3$ \\
\hline OHSU only & $-377.0(+/-25.9)$ & $32.4(+/-0.99)$ & & & $+/-130 \mathrm{~cm}^{\wedge} 3$ \\
\hline Utah only & $-367.7(+/-23.6)$ & $32.1(+/-0.91)$ & & & $+/-113 \mathrm{~cm}^{\wedge} 3$ \\
\hline SA pregnancies & $-383.0(+/-53.5)$ & $33.1(+/-2.18)$ & & & $+/-158 \mathrm{~cm}^{\wedge} 3$ \\
\hline PA pregnancies & $-360.5(+/-35.7)$ & $30.0(+/-1.40)$ & & & $+/-132 \mathrm{~cm}^{\wedge} 3$ \\
\hline Median T1 (ms) & \multicolumn{4}{|c|}{$T_{1}(t)=p_{1}+p_{2} t$} & \\
\hline UN pregnancies & --- & --- & & & \\
\hline OHSU only & $2513.3(+/-49.3)$ & $-26.9(+/-1.88)$ & & & $+/-208 \mathrm{~ms}$ \\
\hline Utah only & --- & --- & & & \\
\hline SA pregnancies & $2555.7(+/-83.0)$ & $-26.5(+/-3.37)$ & & & $+/-158 \mathrm{~ms}$ \\
\hline PA pregnancies & $2591.2(+/-102.0)$ & $-31.2(+/-3.87)$ & & & $+/-246 \mathrm{~ms}$ \\
\hline
\end{tabular}

Table 3. Model fit functions and parameters. Functional forms used for model regression to measured data are indicated for the various data sets described in the text are indicated, along with best-fit model parameter values and parameter uncertainties, and RMS values for model fit residuals (rightmost column). All times $(t)$ are in gestational weeks. 
References 1. Hennington BS, Alexander BT. Linking intrauterine growth restriction and blood pressure: insight into the human origins of cardiovascular disease. Circulation. 2013;128(20):2179-80. 2. Jaddoe VW, de Jonge LL, Hofman A, Franco OH, Steegers EA, Gaillard R. First trimester fetal growth restriction and cardiovascular risk factors in school age children: population based cohort study. BMJ. 2014;348:g14.

3. Kovo M, Schreiber L, Bar J. Placental vascular pathology as a mechanism of disease in pregnancy complications. Thromb Res. 2013;131 Suppl 1:S18-21.

4. Kovo M, Schreiber L, Ben-Haroush A, Gold E, Golan A, Bar J. The placental component in earlyonset and late-onset preeclampsia in relation to fetal growth restriction. Prenat Diagn. 2012;32(7):6327. 5. Paranjothy S, Dunstan F, Watkins WJ, Hyatt M, Demmler JC, Lyons RA, et al. Gestational age, birth weight, and risk of respiratory hospital admission in childhood. Pediatrics. 2013;132(6):e1562-9. 6. Sehgal A, Doctor T, Menahem S. Cardiac function and arterial biophysical properties in small for gestational age infants: postnatal manifestations of fetal programming. J Pediatr. 2013;163(5):1296-300. 7. Walker CK, Krakowiak P, Baker A, Hansen RL, Ozonoff S, Hertz-Picciotto I. Preeclampsia, placental insufficiency, and autism spectrum disorder or developmental delay. JAMA Pediatr. 2015;169(2):154-62.

8. Bar J, Schreiber L, Ben-Haroush A, Ahmed H, Golan A, Kovo M. The placental vascular component in early and late intrauterine fetal death. Thromb Res. 2012;130(6):901-5.

9. Cruz-Lemini M, Crispi F, Van Mieghem T, Pedraza D, Cruz-Martinez R, Acosta-Rojas R, et al. Risk of perinatal death in early-onset intrauterine growth restriction according to gestational age and cardiovascular Doppler indices: a multicenter study. Fetal Diagn Ther. 2012;32(1-2):116-22.

10. Dicke JM, Huettner P, Yan S, Odibo A, Kraus FT. Umbilical artery Doppler indices in small for gestational age fetuses: correlation with adverse outcomes and placental abnormalities. J Ultrasound Med. 2009;28(12):1603-10.

11. Kidron D, Bernheim J, Aviram R. Placental findings contributing to fetal death, a study of 120 stillbirths between 23 and 40 weeks gestation. Placenta. 2009;30(8):700-4.

12. Pare E, Parry S, McElrath TF, Pucci D, Newton A, Lim KH. Clinical risk factors for preeclampsia in the 21st century. Obstet Gynecol. 2014;124(4):763-70.

13. Roberts DJ, Post MD. The placenta in pre-eclampsia and intrauterine growth restriction. J Clin Pathol. 2008;61(12):1254-60.

14. Salafia CM, Vintzileos AM, Silberman L, Bantham KF, Vogel CA. Placental pathology of idiopathic intrauterine growth retardation at term. Am J Perinatol. 1992;9(3):179-84.

15. Salafia CM, Vogel CA, Bantham KF, Vintzileos AM, Pezzullo J, Silberman L. Preterm delivery: correlations of fetal growth and placental pathology. Am J Perinatol. 1992;9(3):190-3.

16. Sibai B, Dekker G, Kupferminc M. Pre-eclampsia. Lancet. 2005;365(9461):785-99.

17. Stillbirth Collaborative Research Network Writing G. Association between stillbirth and risk factors known at pregnancy confirmation. JAMA. 2011;306(22):2469-79.

18. Bell AW, Hay WW, Jr., Ehrhardt RA. Placental transport of nutrients and its implications for fetal growth. J Reprod Fertil Suppl. 1999;54:401-10.

19. Gude NM, Roberts CT, Kalionis B, King RG. Growth and function of the normal human placenta. Thromb Res. 2004;114(5-6):397-407.

20. Murphy VE, Smith R, Giles WB, Clifton VL. Endocrine regulation of human fetal growth: the role of the mother, placenta, and fetus. Endocr Rev. 2006;27(2):141-69.

21. Ozkaya U, Ozkan S, Ozeren S, Corakci A. Doppler examination of uteroplacental circulation in early pregnancy: can it predict adverse outcome? J Clin Ultrasound. 2007;35(7):382-6. 
22. Poon LC, Lesmes C, Gallo DM, Akolekar R, Nicolaides KH. Prediction of small-for-gestational-age neonates: screening by biophysical and biochemical markers at 19-24 weeks. Ultrasound Obstet Gynecol. 2015;46(4):437-45.

23. Su EJ. Role of the fetoplacental endothelium in fetal growth restriction with abnormal umbilical artery Doppler velocimetry. Am J Obstet Gynecol. 2015;213(4 Suppl):S123-30.

24. Verlohren S, Melchiorre K, Khalil A, Thilaganathan B. Uterine artery Doppler, birth weight and timing of onset of pre-eclampsia: providing insights into the dual etiology of late-onset pre-eclampsia. Ultrasound Obstet Gynecol. 2014;44(3):293-8.

25. Zhong $\mathrm{Y}$, Tuuli M, Odibo AO. First-trimester assessment of placenta function and the prediction of preeclampsia and intrauterine growth restriction. Prenat Diagn. 2010;30(4):293-308.

26. Tuuli MG, Odibo AO. The role of serum markers and uterine artery Doppler in identifying at-risk pregnancies. Clin Perinatol. 2011;38(1):1-19, v.

27. Mitra SC, Seshan SV, Riachi LE. Placental vessel morphometry in growth retardation and increased resistance of the umbilical artery Doppler flow. J Matern Fetal Med. 2000;9(5):282-6.

28. O'Dwyer V, Burke G, Unterscheider J, Daly S, Geary MP, Kennelly MM, et al. Defining the residual risk of adverse perinatal outcome in growth-restricted fetuses with normal umbilical artery blood flow. Am J Obstet Gynecol. 2014;211(4):420 e1-5.

29. Unterscheider J, Daly S, Geary MP, Kennelly MM, McAuliffe FM, O'Donoghue K, et al. Optimizing the definition of intrauterine growth restriction: the multicenter prospective PORTO Study. Am J Obstet Gynecol. 2013;208(4):290 e1-6.

30. Guttmacher AE, Maddox YT, Spong CY. The Human Placenta Project: placental structure, development, and function in real time. Placenta. 2014;35(5):303-4.

31. Sorensen A, Peters D, Frund E, Lingman G, Christiansen O, Uldbjerg N. Changes in human placental oxygenation during maternal hyperoxia estimated by blood oxygen level-dependent magnetic resonance imaging (BOLD MRI). Ultrasound Obstet Gynecol. 2013;42(3):310-4.

32. Ogawa $S$, Lee TM, Kay AR, Tank DW. Brain magnetic resonance imaging with contrast dependent on blood oxygenation. Proc Natl Acad Sci U S A. 1990;87(24):9868-72.

33. Frias $\mathrm{AE}$, Schabel MC, Roberts VH, Tudorica A, Grigsby PL, Oh KY, et al. Using dynamic contrastenhanced $\mathrm{MRI}$ to quantitatively characterize maternal vascular organization in the primate placenta. Magn Reson Med. 2015;73(4):1570-8.

34. Schabel MC, Roberts VHJ, Lo JO, Platt S, Grant KA, Frias AE, et al. Functional imaging of the nonhuman primate Placenta with endogenous blood oxygen level-dependent contrast. Magn Reson Med. 2016;76(5):1551-62.

35. Hirsch AJ, Roberts VHJ, Grigsby PL, Haese N, Schabel MC, Wang X, et al. Zika virus infection in pregnant rhesus macaques causes placental dysfunction and immunopathology. Nat Commun. 2018;9(1):263.

36. Lo JO, Roberts VHJ, Schabel MC, Wang X, Morgan TK, Liu Z, et al. Novel Detection of Placental Insufficiency by Magnetic Resonance Imaging in the Nonhuman Primate. Reprod Sci. 2018;25(1):64-73. 37. Lo JO, Schabel MC, Roberts VH, Wang X, Lewandowski KS, Grant KA, et al. First trimester alcohol exposure alters placental perfusion and fetal oxygen availability affecting fetal growth and development in a non-human primate model. Am J Obstet Gynecol. 2017;216(3):302 e1- e8.

38. Anderson KB, Andersen AS, Hansen DN, Sinding M, Peters DA, Frokjaer JB, et al. Placental transverse relaxation time (T2) estimated by MRI: Normal values and the correlation with birthweight. Acta Obstet Gynecol Scand. 2020.

39. Hutter J, Slator PJ, Jackson L, Gomes ADS, Ho A, Story L, et al. Multi-modal functional MRI to explore placental function over gestation. Magn Reson Med. 2019;81(2):1191-204.

40. Melbourne A, Aughwane R, Sokolska M, Owen D, Kendall G, Flouri D, et al. Separating fetal and maternal placenta circulations using multiparametric MRI. Magn Reson Med. 2019;81(1):350-61. 
41. Nye GA, Ingram E, Johnstone ED, Jensen OE, Schneider H, Lewis RM, et al. Human placental oxygenation in late gestation: experimental and theoretical approaches. J Physiol. 2018;596(23):5523-

73334.

42. Sinding M, Peters DA, Poulsen SS, Frokjaer JB, Christiansen OB, Petersen A, et al. Placental baseline conditions modulate the hyperoxic BOLD-MRI response. Placenta. 2018;61:17-23. 43. Schabel MC, Roberts VHJ, Rincon DM, Gaffney JE, Lo JO, Gibbins KJ, et al. A Longitudinal Multisite Study of Endogenous BOLD MRI In Human Pregnancies. Magnetic Resonance in Medicine. 2019:1075.

44. You W, Andescavage NN, Kapse K, Donofrio MT, Jacobs M, Limperopoulos C. Hemodynamic Responses of the Placenta and Brain to Maternal Hyperoxia in Fetuses with Congenital Heart Disease by Using Blood Oxygen-Level Dependent MRI. Radiology. 2020;294(1):141-8. 45. Oken E, Kleinman KP, Rich-Edwards J, Gillman MW. A nearly continuous measure of birth weight for gestational age using a United States national reference. BMC Pediatr. 2003;3:6. magnetic resonance imaging from first trimester to term gestation. Pediatr Radiol. 2018;48(13):1936-44. 47. Oh KY, Roberts VH, Schabel MC, Grove KL, Woods M, Frias AE. Gadolinium Chelate Contrast Material in Pregnancy: Fetal Biodistribution in the Nonhuman Primate. Radiology. 2015;276(1):110-8. 48. Prola-Netto J, Woods M, Roberts VHJ, Sullivan EL, Miller CA, Frias AE, et al. Gadolinium Chelate Safety in Pregnancy: Barely Detectable Gadolinium Levels in the Juvenile Nonhuman Primate after in 750 Utero Exposure. Radiology. 2018;286(1):122-8.

751 49. Gestational Hypertension and Preeclampsia: ACOG Practice Bulletin, Number 222. Obstet 752 Gynecol. 2020;135(6):e237-e60.

753 50. Gibbins KJ, Pinar H, Reddy UM, Saade GR, Goldenberg RL, Dudley DJ, et al. Findings in Stillbirths 754 Associated with Placental Disease. Am J Perinatol. 2020;37(7):708-15.

755 51. Spinillo A, Gardella B, Bariselli S, Alfei A, Silini E, Dal Bello B. Placental histopathological correlates of umbilical artery Doppler velocimetry in pregnancies complicated by fetal growth restriction. Prenat Diagn. 2012;32(13):1263-72.

758 52. Mayhew TM. Patterns of villous and intervillous space growth in human placentas from normal 759 and abnormal pregnancies. Eur J Obstet Gynecol Reprod Biol. 1996;68(1-2):75-82. 

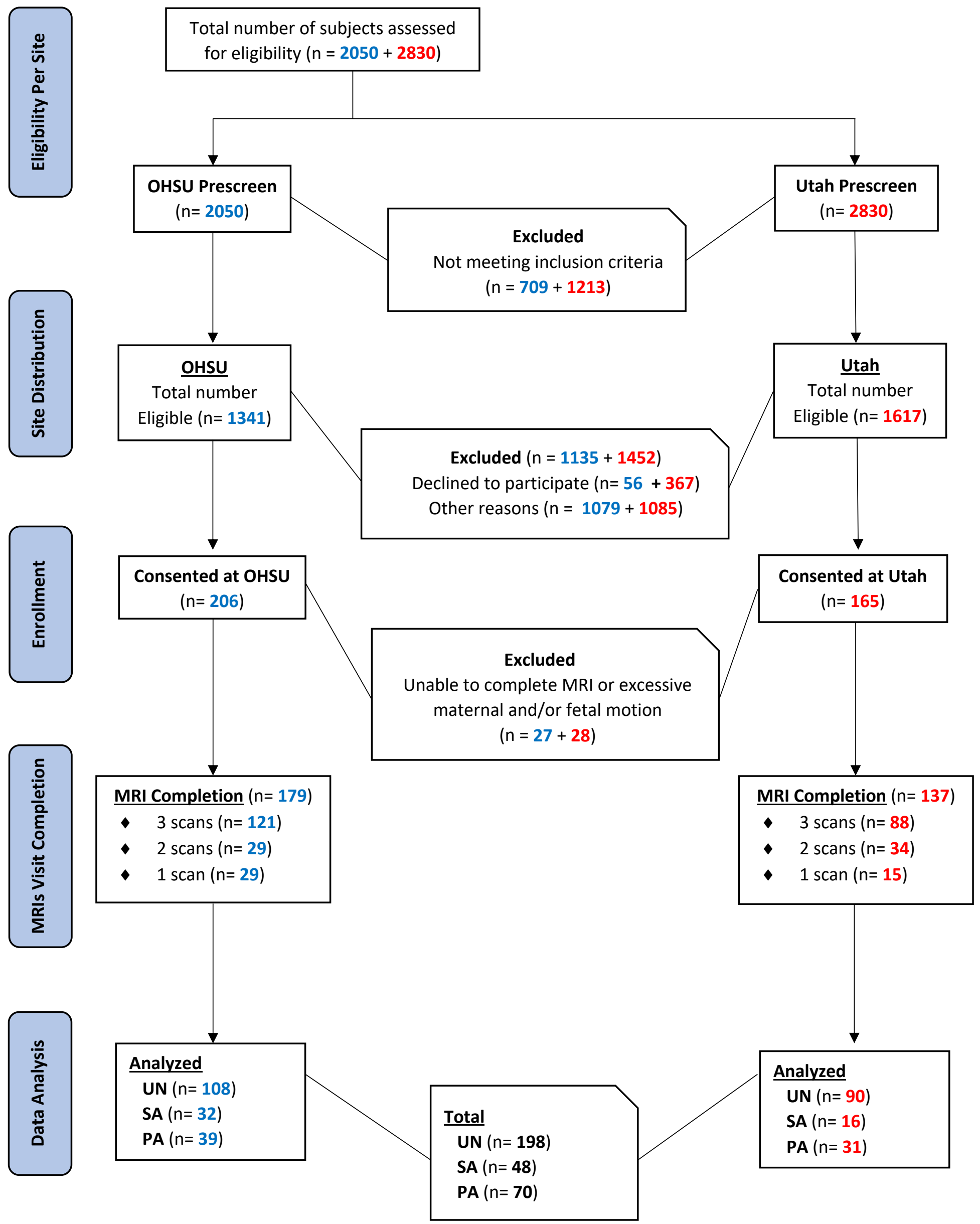

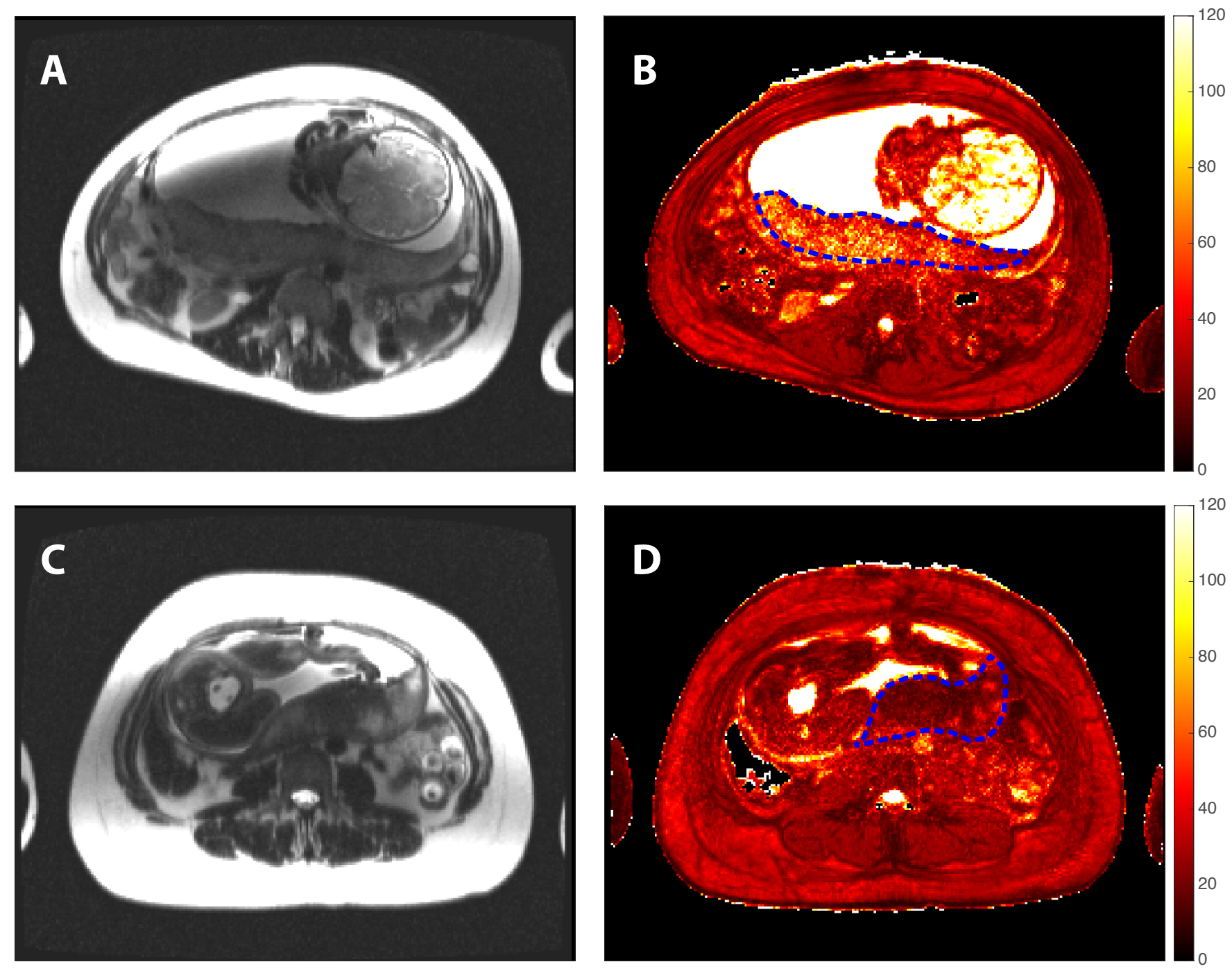

Figure 2 

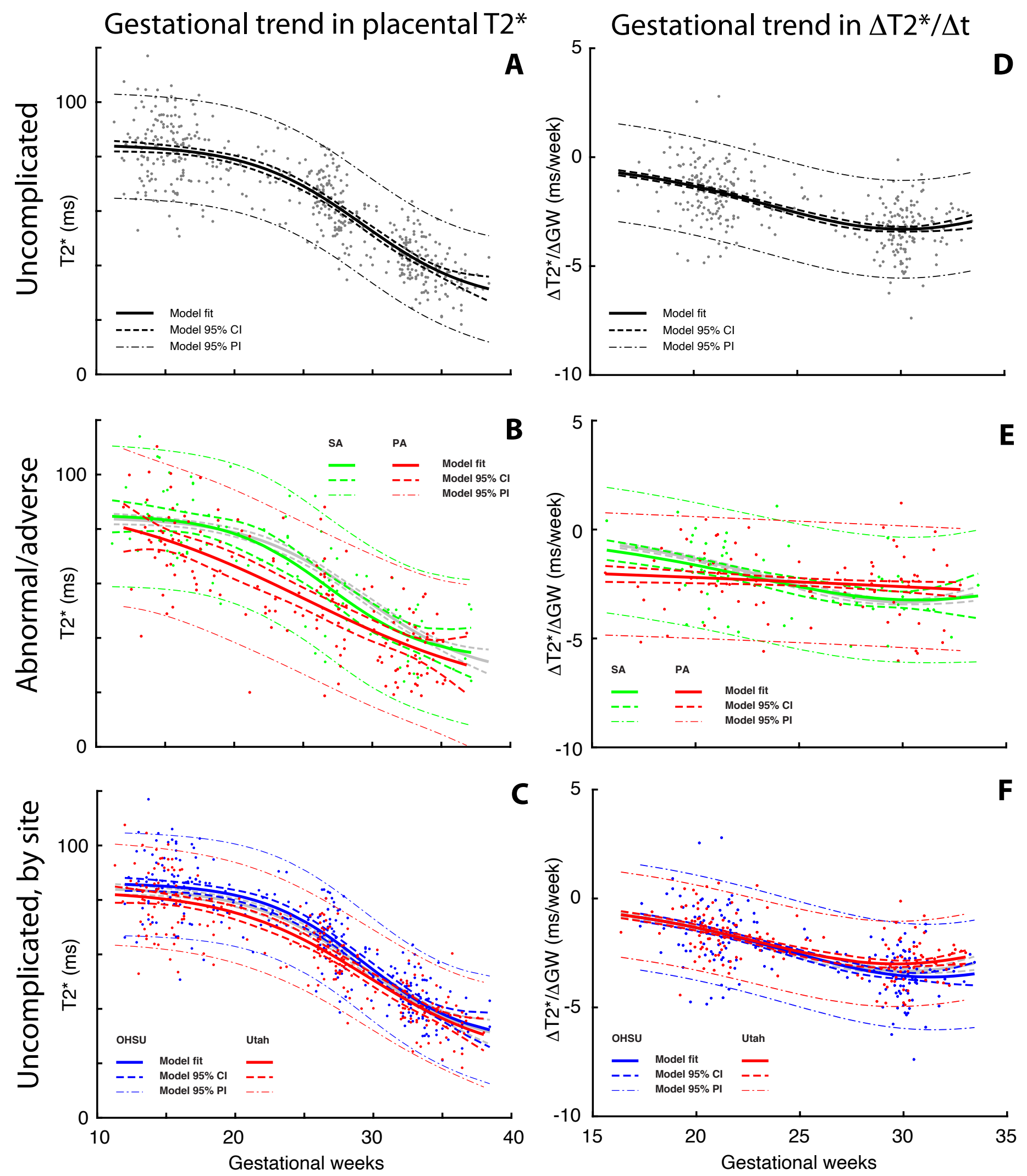

Figure 3 


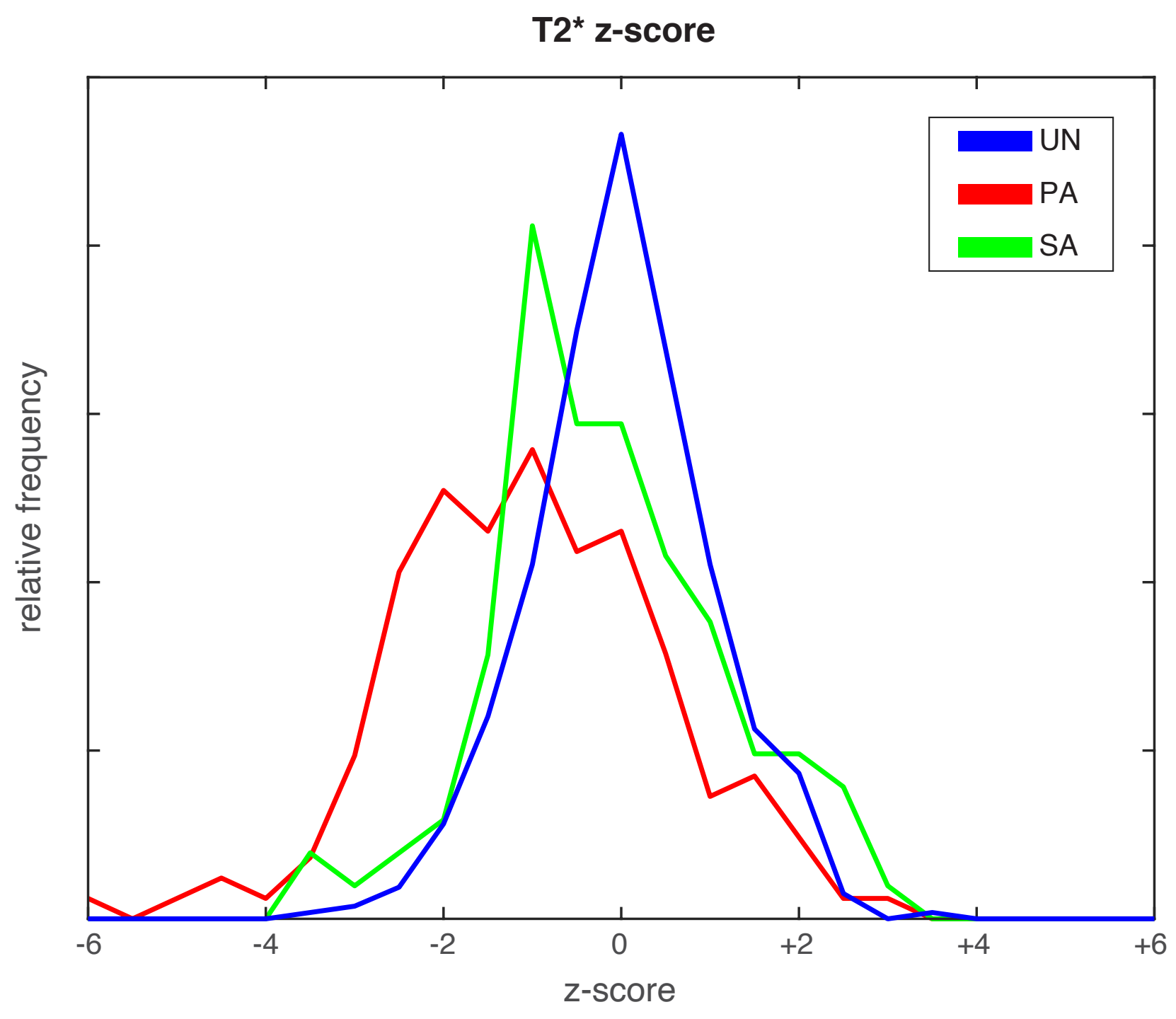

Figure 4 


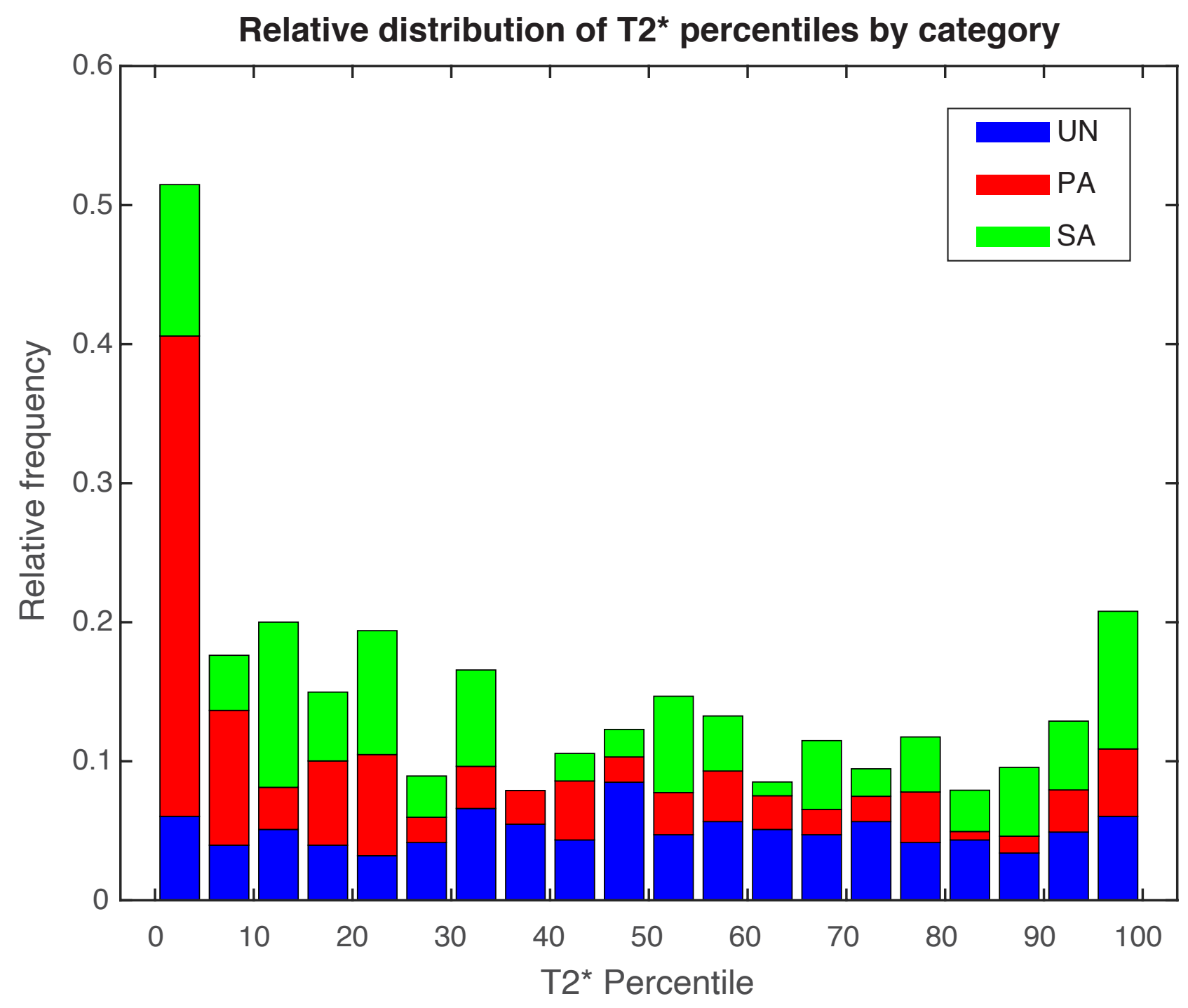

Figure 5 

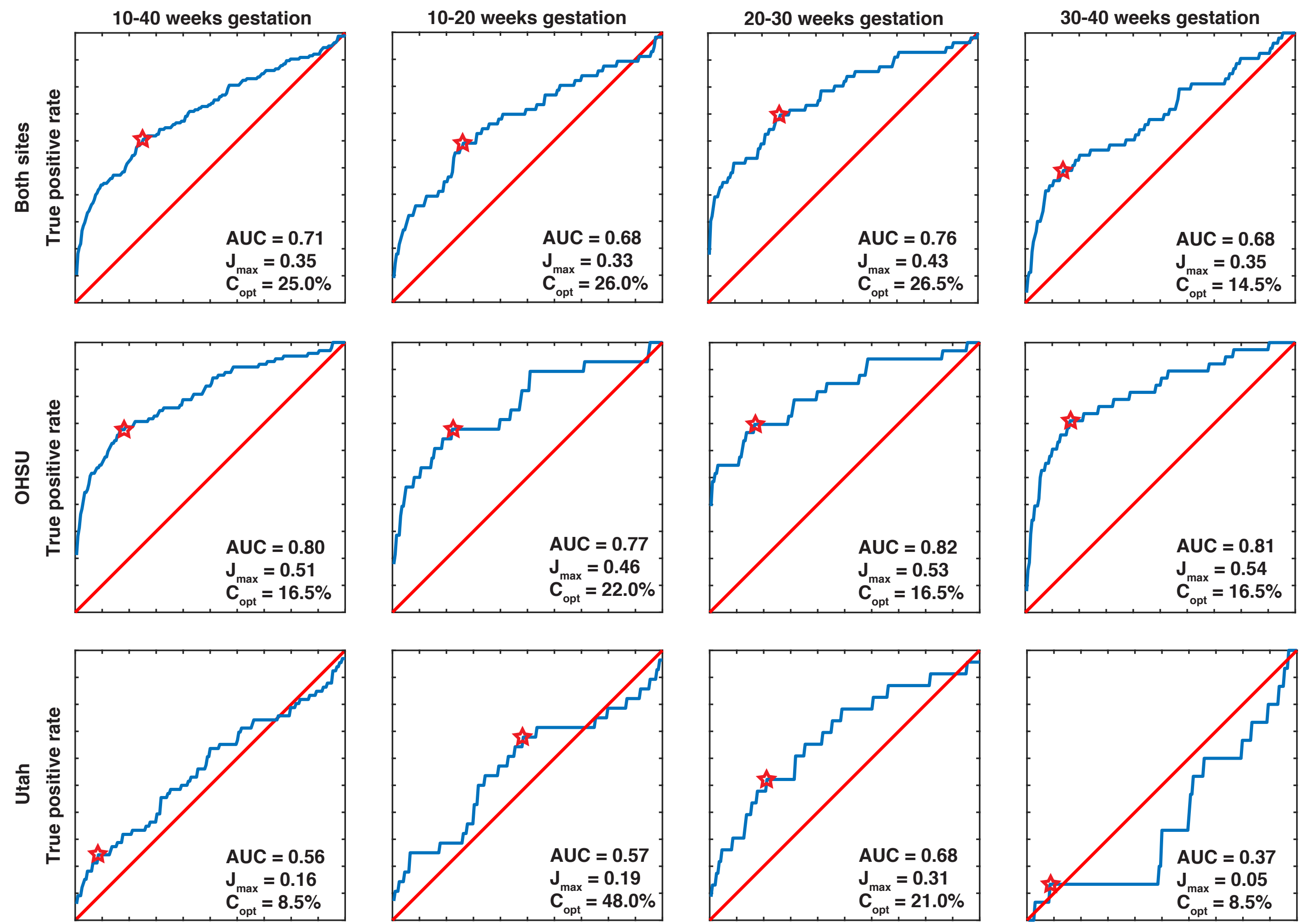

False positive rate

False positive rate

False positive rate

False positive rate

Figure 6 


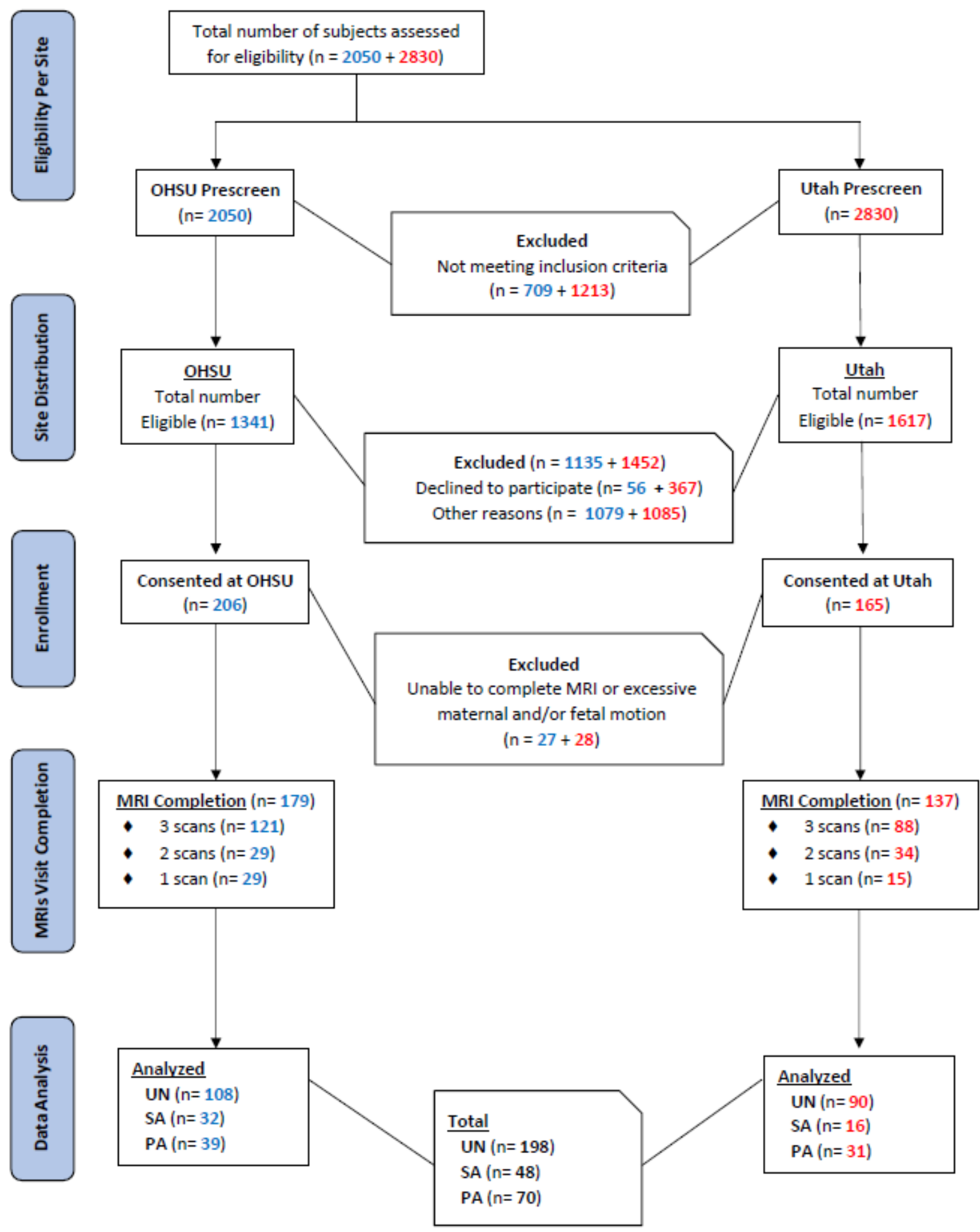

Figure 1

Enrollment flow chart. A flow chart detailing numbers of prospective patients screened, consented, and enrolled at both study sites, along with numbers of completed MRI studies meeting quality criteria for inclusion in data analysis presented here. 

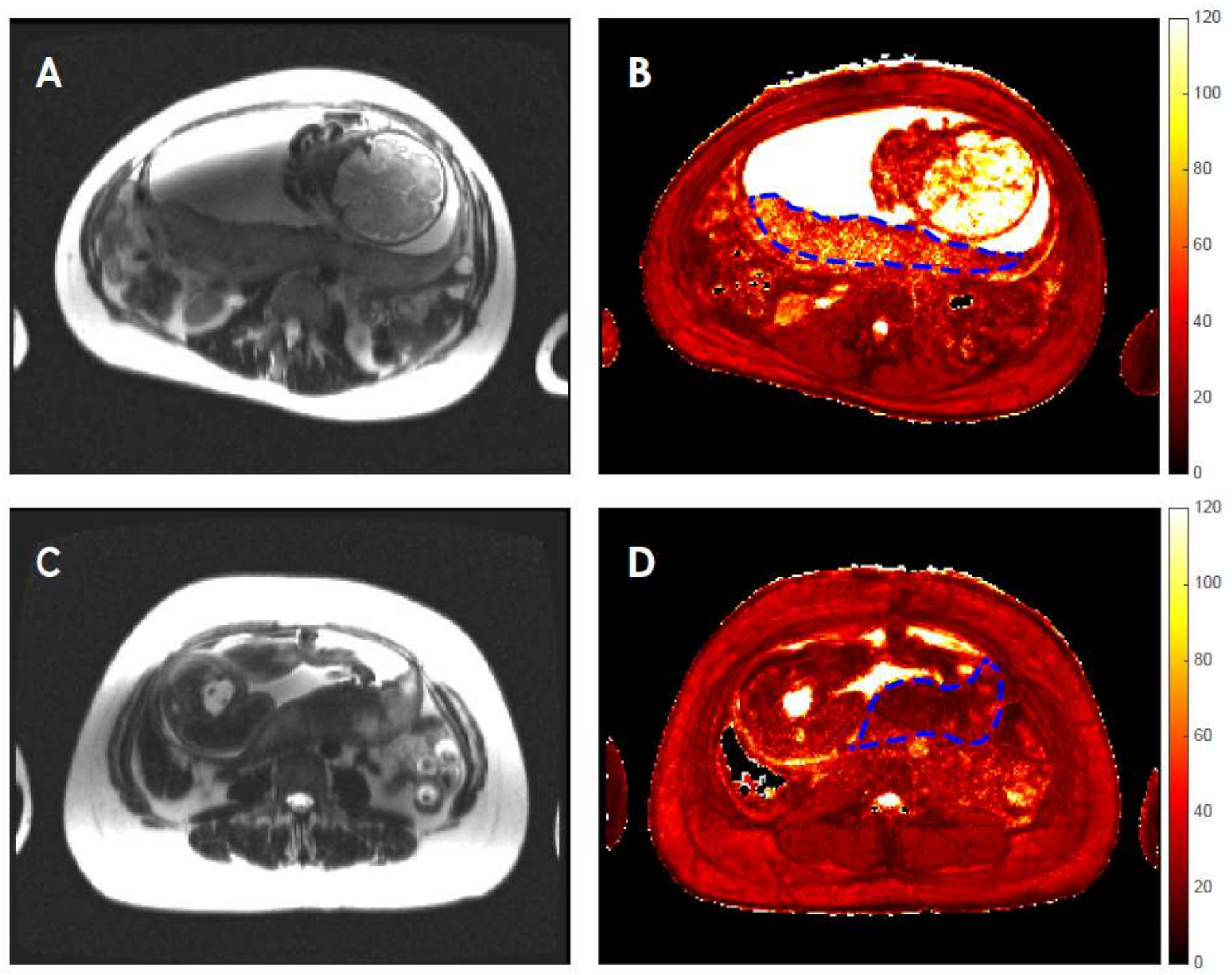

Figure 2

Comparison of anatomic imaging (T2-weighted HASTE, left column) and placental T2* mapping (right column) in a uncomplicated normal pregnancy at 232 days gestation (top row, panels A \& B) with those from a primary adverse pregnancy at 235 days gestation presenting with severe preeclampsia (bottom row, panels $C \& D$ ). The placenta is indicated by the dashed blue outlines overlaid on the T2* maps. 

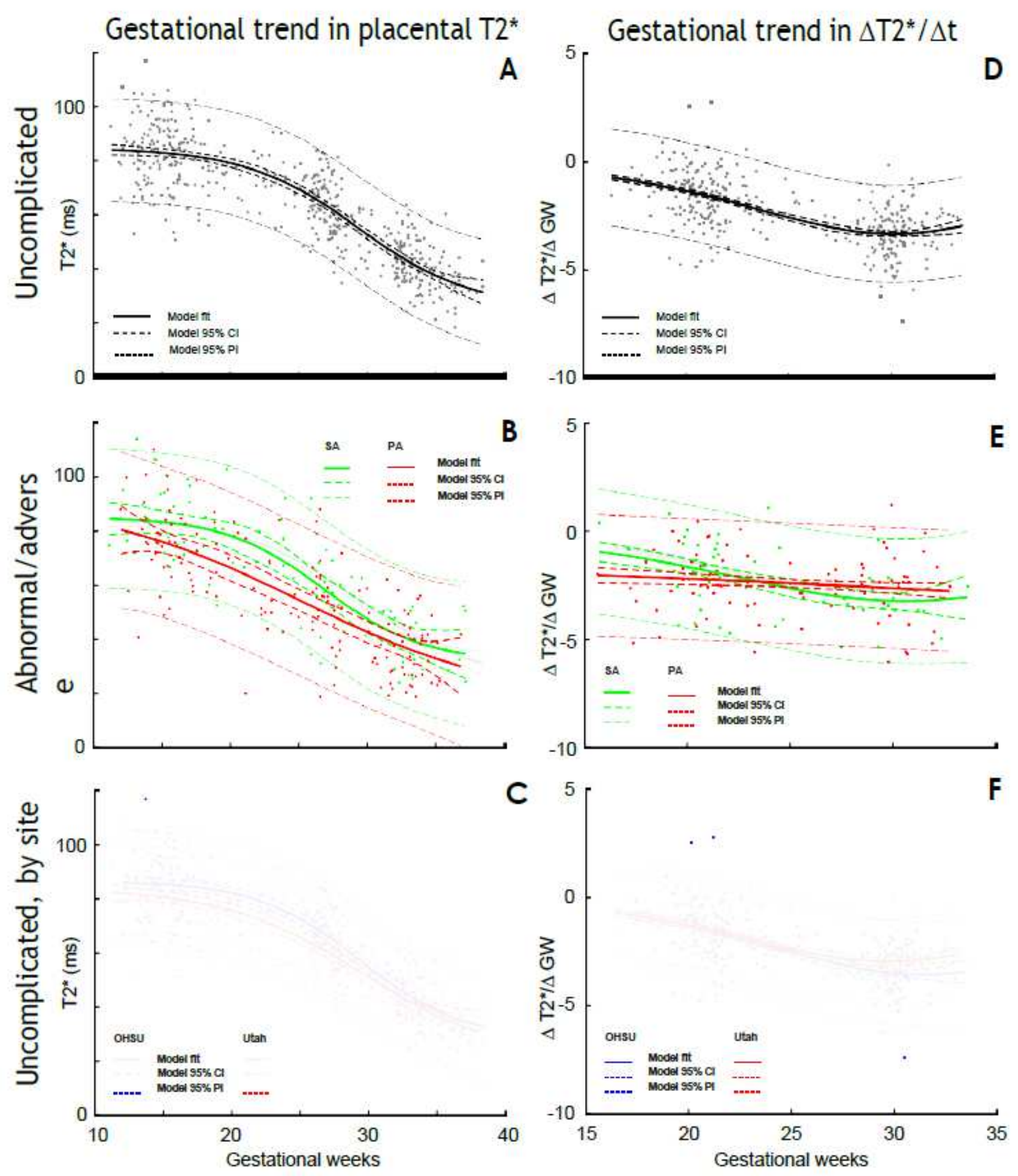

\section{Figure 3}

Gestational dependence of placental T2* values. Median T2* values for each completed study, computed over the entire placenta, are plotted as a function of gestational age at time of imaging in the three panels in the left column (panels $A, B, C$ ), while corresponding rates of change in placental T2* between repeated imaging time points within the same individual are plotted as a function of gestational age in the right column (panels D, E, F). The upper row plots these quantities for normal pregnancies, the middle row for 
abnormal (green) and adverse (red) pregnancies, and the bottom row for normal pregnancies stratified by site (OHSU in blue, Utah in red). In all graphs, model regression curves (using the functions defined in Table 2) are indicated by the thick solid lines, the $95 \%$ confidence intervals by the dashed lines, and the $95 \%$ prediction intervals by the dot-dashed lines. The best fit and $95 \% \mathrm{Cl}$ curves from the plots in the upper row are shown in gray in the middle and bottom rows for reference.

\section{T2* Z-score}

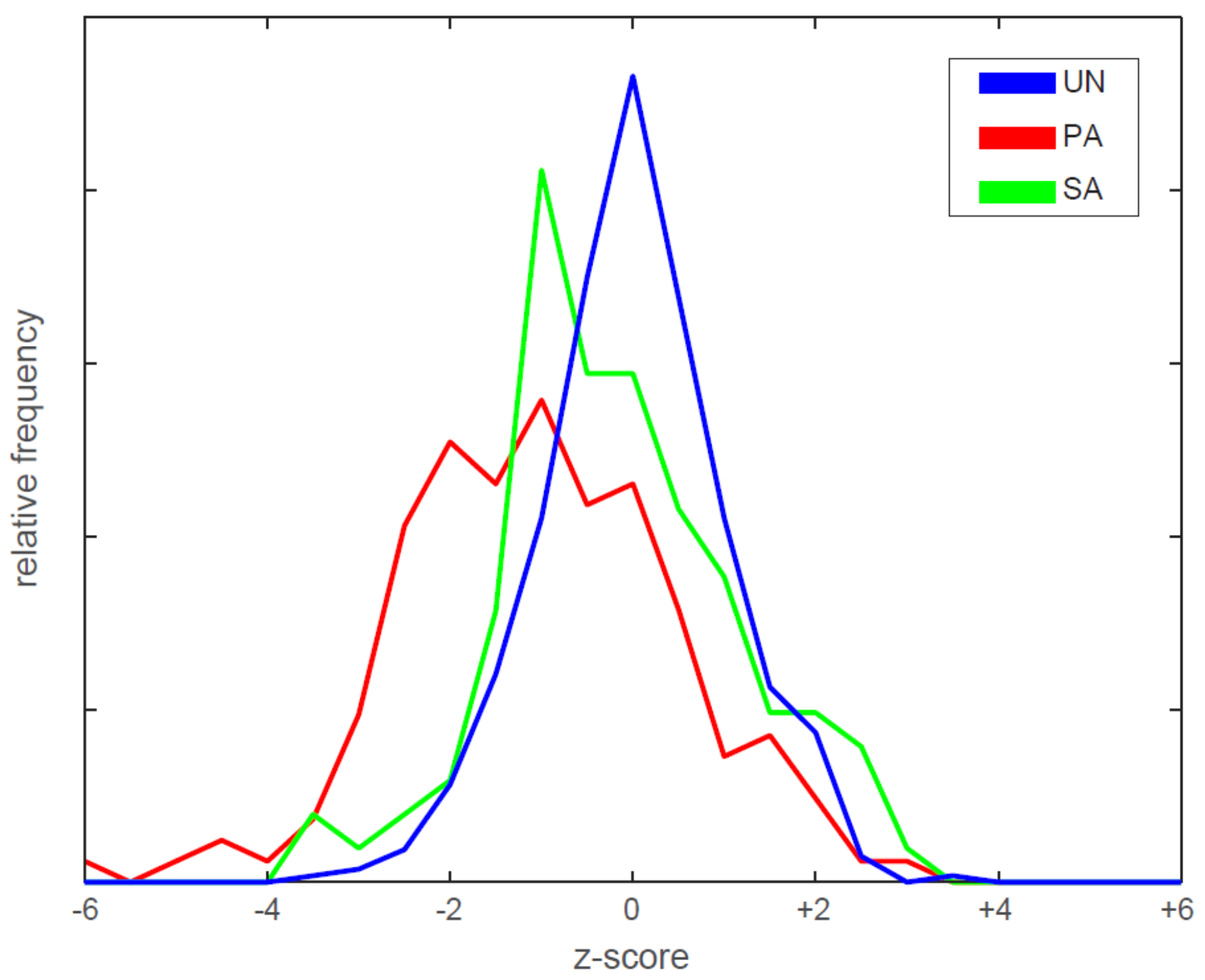

\section{Figure 4}

Histograms of T2* Z-scores in normal, abnormal, and adverse pregnancies. Z-score histograms shown are computed using prediction intervals for sigmoid model regression to T2* measurements in UN pregnancies, applied to individual studies in UN (blue), PA (red), and SA (green) pregnancies. 


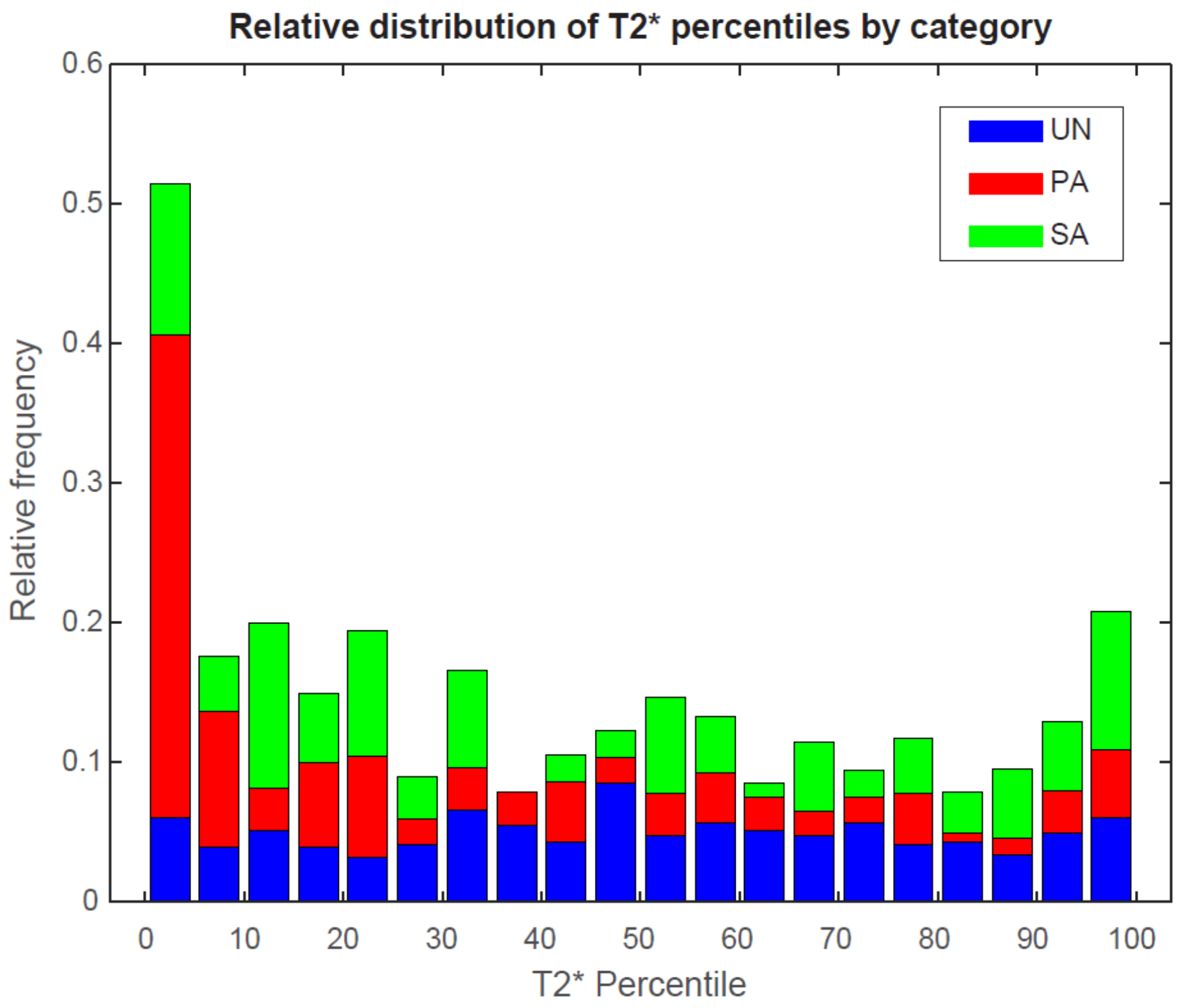

Figure 5

Bar chart of distribution of measured T2* percentiles for uncomplicated normal, primary adverse, and secondary abnormal pregnancies. 

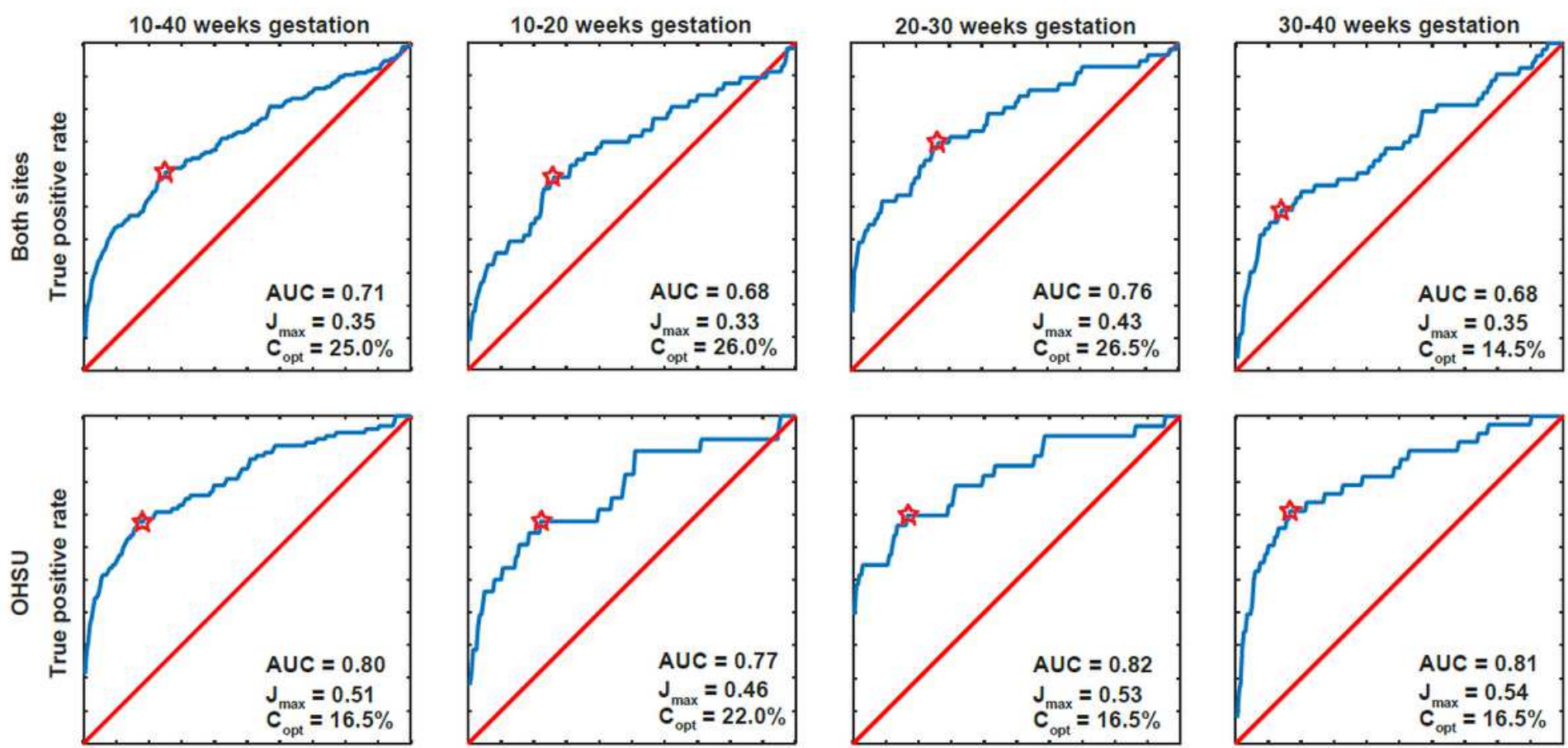

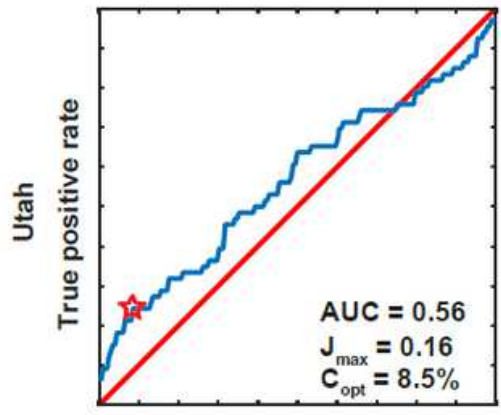

False positive rate

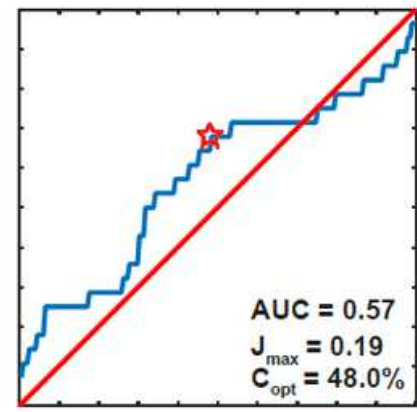

False positive rate

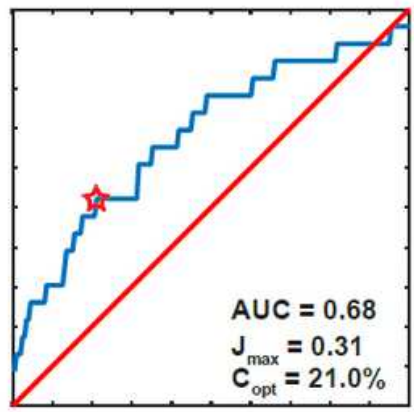

False positive rate

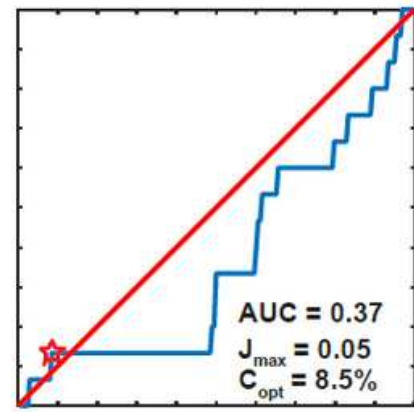

False positive rate

Figure 6

Receiver operator characteristic (ROC) curves for T2* measurements in pregnancies with our primary adverse outcome relative to uncomplicated normal pregnancies. The points where Youden's $\mathrm{J}$ is maximized are indicated by the stars. Area under the curve (AUC), $\mathrm{J}$ max, and the corresponding optimal cutoff threshold in ${ }^{2}{ }^{*}$ percentile relative to UN (C opt) are given in the figure legend for each panel. 